\title{
Management of Peritoneal Malignancies
}

\author{
Richard N. Berri and Jennifer M. Ford
}

\section{Introduction}

Peritoneal malignancies may result in a widespread disease process, peritoneal carcinomatosis (PC), which has significant morbidity and mortality for patients afflicted by this disease. Dissemination into the peritoneum and throughout the abdomen can be due to a primary peritoneal cancer or other primary malignancies that have metastasized, including (but not limited to) colorectal cancer, gastric cancer, pancreatic cancer, appendiceal cancer, ovarian cancer, and mesothelioma. Patients with gastrointestinal (GI) or gynecologic malignancies with peritoneal carcinomatosis may have dismal survival due to a high disease burden within the abdominal cavity [1]. Some studies suggest the average survival for patients with peritoneal carcinomatosis of colorectal origin is 18-48 months, for high-grade appendiceal adenocarcinoma 12-36 months, and for low-grade appendiceal neoplasms $>60$ months [1].

As the understanding of peritoneal malignancies and peritoneal carcinomatosis evolved, it may now be acceptable to treat this as locoregional disease [2]. Dr. Paul Sugarbaker, a pioneer in the management of peritoneal cancer, was instrumental in this paradigm shift and his emphasis on accurate assessment of the locoregional tumor burden helped develop the current treatment pathways that are followed today. Cytoreductive surgery (CRS) plus hyperthermic intraperitoneal chemotherapy (HIPEC) is now the accepted treatment for PC in select patients with acceptable disease burden from particular malignancies and a good functional status

R. N. Berri $(\bowtie)$

Surgical Oncology, Peritoneal Malignancies Program, Department of Surgery, Ascension, St. John Hospital,

Grosse Pointe, MI, USA

e-mail: Richard.Berri@ascension.org

J. M. Ford

Department of Surgery, Astria Regional Medical Center and Heart Institute, Yakima, WA, USA
Table 23.1 Comparing survival rates with CRS + HIPEC versus chemotherapy (CT) alone

\begin{tabular}{l|l|l|l}
\hline & & & $\begin{array}{l}\text { Mean survival } \\
\text { (months) }\end{array}$ \\
\hline Author/study & Disease & CRS + HIPEC & CT alone \\
\hline $\begin{array}{l}\text { Netherlands } \\
\text { Cancer }\end{array}$ & $\begin{array}{l}\text { Colorectal with } \\
\text { peritoneal } \\
\text { metastasis } \\
\text { or + cytology }\end{array}$ & 22.2 & 12.6 \\
\hline Elias et al. [4] & $\begin{array}{l}\text { Colorectal with } \\
\text { peritoneal } \\
\text { metastasis }\end{array}$ & 62.7 & 23.9 \\
\hline Glehen et al. [5] & Colorectal cancer & 19.2 & - \\
\hline
\end{tabular}

(Table 23.1) [3-5]. The ultimate goal of HIPEC is to destroy microscopic disease left behind after optimal CRS. As outlined by the American Society of Peritoneal Surface Malignancies, indications for CRS with HIPEC are as follows: a large volume of noninvasive peritoneal carcinomatosis or sarcomatosis, peritoneal mesothelioma, low-volume peritoneal seeding from invasive cancer, perforated GI cancer, cancer adherent to adjacent organs or structures, GI cancer with positive peritoneal cytology, GI with ovarian involvement, intraoperative tumor spill, or after systemic chemotherapy for recurrent ovarian cancer after a long disease-free interval and for palliation of patients with malignant ascites [6].

\section{Patient Selection and Diagnosis}

Perhaps the most important factor in this disease entity is patient selection and establishing which patients may benefit from a surgical approach. This can be extremely challenging. Patients who present for evaluation need a comprehensive workup to establish a diagnosis and determine the extent of disease. Ideally, patients should be referred to high-volume centers that have extensive experience in diagnosing and treating peritoneal malignancies. A detailed physical examination and history, including all previous treatments and 
Table 23.2 The Eastern Cooperative Oncology Group (ECOG) Performance Status categories [7]

\begin{tabular}{l|l}
\hline Grade & Performance status \\
\hline 0 & $\begin{array}{l}\text { Fully active, able to carry on all pre-disease performance } \\
\text { without restriction }\end{array}$ \\
\hline 1 & $\begin{array}{l}\text { Restricted in physically strenuous activity, but ambulatory } \\
\text { and able to carry out work of a light or sedentary nature, } \\
\text { e.g., light house work, office work }\end{array}$ \\
\hline 2 & $\begin{array}{l}\text { Ambulatory and capable of all self-care, but unable to carry } \\
\text { out any work activities; up and about more than 50\% of } \\
\text { waking hours }\end{array}$ \\
\hline 3 & $\begin{array}{l}\text { Capable of only limited self-care; confined to bed or chair } \\
\text { more than 50\% of waking hours }\end{array}$ \\
\hline 4 & $\begin{array}{l}\text { Completely disabled; cannot carry on any self-care; totally } \\
\text { confined to bed or chair }\end{array}$ \\
\hline 5 & Dead \\
\hline
\end{tabular}

when they occurred, should be established during the first evaluation. The two factors that will ultimately have the most substantial impact on the outcome of the patient is the histology of the primary tumor and the overall burden of peritoneal disease.

Optimization of preoperative performance status (PS), activity level, and comorbidities cannot be overemphasized. Often employed is the Eastern Cooperative Oncology Group (ECOG) Performance Status; this scale ranges from 0 to 5 and provides a concise method to assess performance status and activity level of patients (see Table 23.2) [7]. Comorbidities should be well controlled both preoperatively and postoperatively. Previously diagnosed comorbidities are present in $18 \%$ of patients undergoing major oncologic resection. These morbidities increase the risk of acute medical complications (odds ratio [OR] 3.7), in-hospital mortality (OR 3.6), hospital costs, postoperative complications (OR 3.9), and increased complication severity (OR 3.6) $[8]$.

The American College of Surgeons (ACS) National Surgical Quality Improvement Program (NSQIP) calculator is a tool that can assist in preoperative evaluation. A recent study from our institution validated the risk calculator for use in this patient population [9]. The risk calculator is a reasonable tool and is now integrated into our preoperative evaluation.

Any patient who demonstrates a poor PS or uncontrolled comorbidities must be optimized prior to considering surgical resection in such a way that is similar to any patient being evaluated for any complicated GI oncologic resection. Patients benefit from a regimen of physical activity, smoking cessation, and medical and nutritional optimization. Additionally, the procedure, recovery, outcomes, and possible adverse effects must all be explained to the patient so they have a comprehensive understanding and not unrealistic expectations.

\section{Radiographic Imaging}

The use of imaging is essential and is in some cases diagnostic of peritoneal carcinomatosis. It is able to reasonably discern those patients that demonstrate hematogenous metastasis outside of the peritoneal cavity and non-resectable liver, lung, or other distant metastasis and are therefore not surgical candidates. However, an important tenant of peritoneal cancer is that any and all imaging modalities may most often dramatically underestimate the true volume and burden of peritoneal carcinomatosis. Importantly, this must be kept in mind in the preoperative surgical planning and especially in discussion with the patient to alert them of this possibility of "understaging" with imaging (Fig. 23.1) [10].

Imaging modalities used include computed tomography (CT), magnetic resonance imaging (MRI), ${ }^{18} \mathrm{~F}$-fluorodeoxyglucose (FDG) positron emission tomography (PET) scan, and ultrasound (US). US can identify the presence of ascites and can be used for image-guided biopsy or identification of large intra-abdominal masses [2]. CT scan or, if available, MRI with peritoneal malignancy protocol is the method most often employed. It can discern size (of nodules), location, type of PC, and possible primary sites (Figs. 23.2 and 23.3) [2].

The use of multiplanar CT image reconstruction helps identify lesions that are small $(<5 \mathrm{~mm})$ or located in difficult to visualize anatomic positions, such as paracolic gutter or hepatorenal space. Smaller nodules $(<5 \mathrm{~mm})$ are better visualized when located on the surface of a larger solid organ, such as the spleen or liver [2]. An extremely valuable fundamental is that the inherent movement of fluid within the abdomen deposits disease first within the right upper quadrant/right subdiaphragmatic peritoneum, followed by the left subphrenic space. In the lower abdomen, the rectovaginal pouch/pouch of Douglas is the initial collection space, followed by deposits around the urinary bladder then the paracolic gutters.

The provisional different types of PC are sclerotic, infiltrative, micronodular/military, and macronodular/nodus. These are often identifiable on CT but much overlap between types exists. Therefore categorization is based on dominant radiographic characteristics. Peritoneal folds appear thickened (diffuse or focal) with sclerotic, jellylike, reticular, reticulonodular, nodular, or large plaques [2].

The sclerotic type often involves the mesentery that appears thickened and retracted [2]. The greater omentum can demonstrate "omental caking," which is a thick heterogeneous neoplastic mixture of micronodular, nodular, or large plaque disease [2]. When calcifications are identified it is often due to the presence of nodular or plaque lesions [2]. These lesions show cyst-like appearances with various levels of attenuation [2]. 

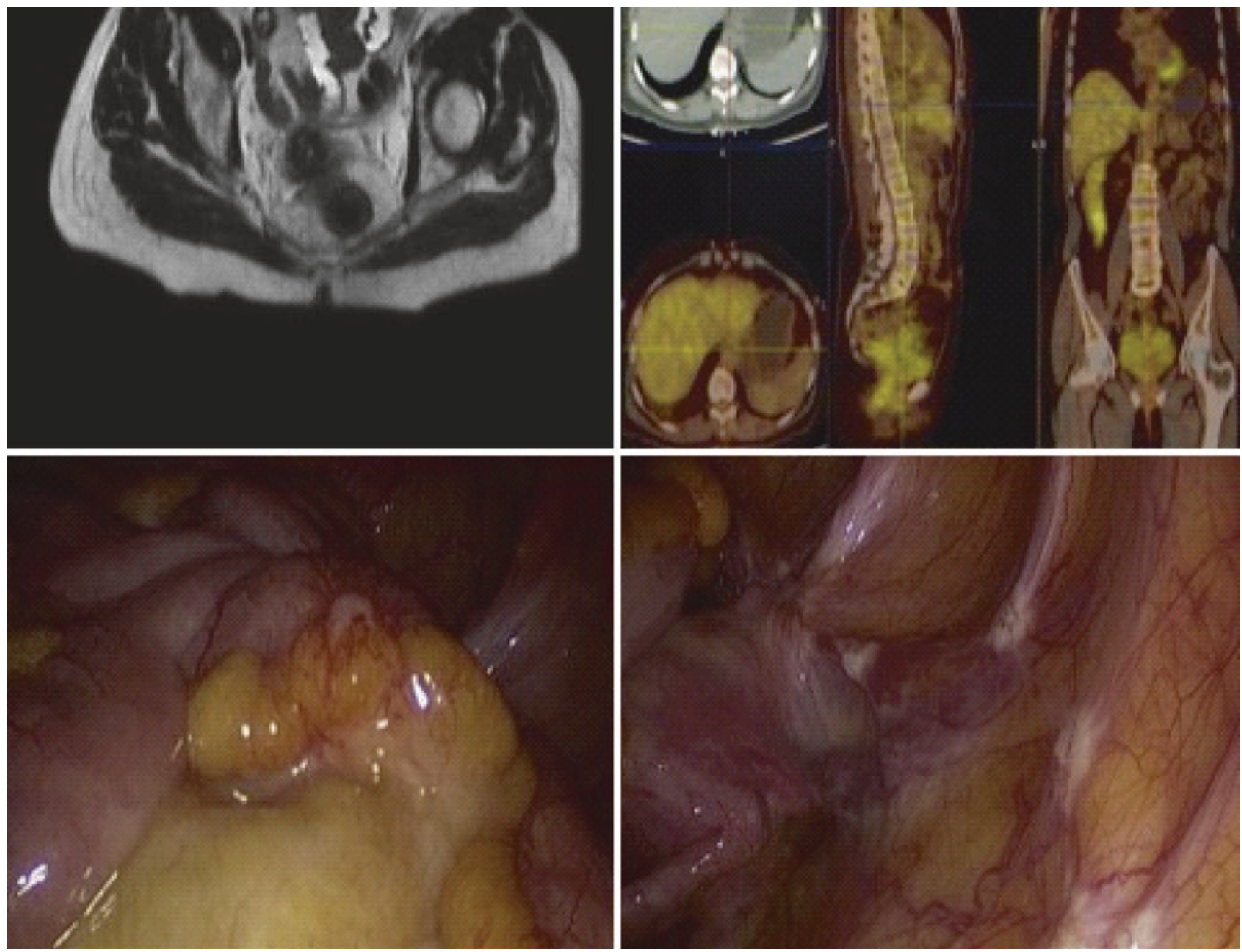

Fig. 23.1 Top left and right: CT, MRI, PET all negative. Bottom (L and R): laparoscopy with gross disease in a patient with colorectal peritoneal carcinomatosis. (Reprinted with permission from Valle et al. [10].)
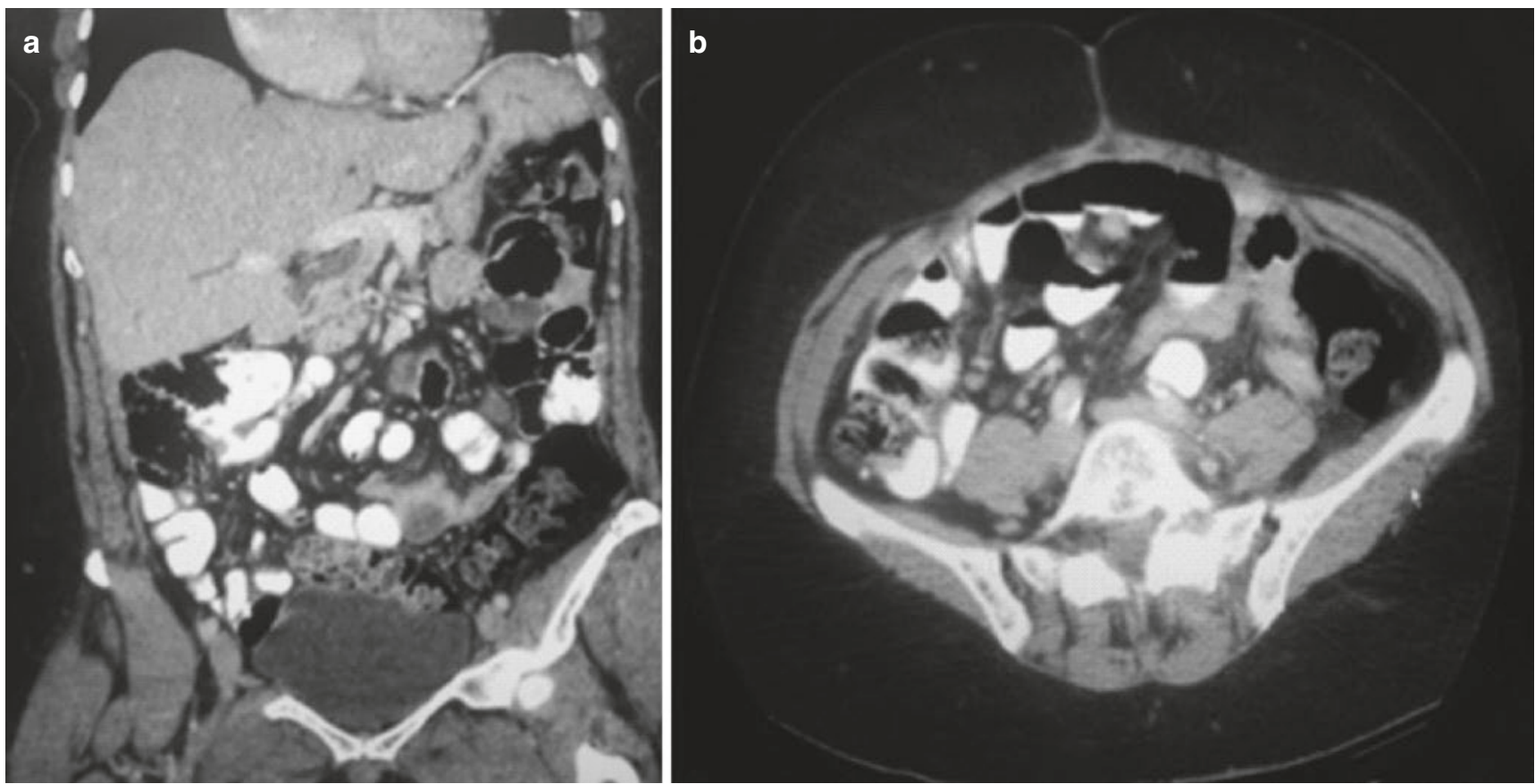

Fig. 23.2 (a, b) A patient's CT scan that contains minimal mucinous ascites and disease 

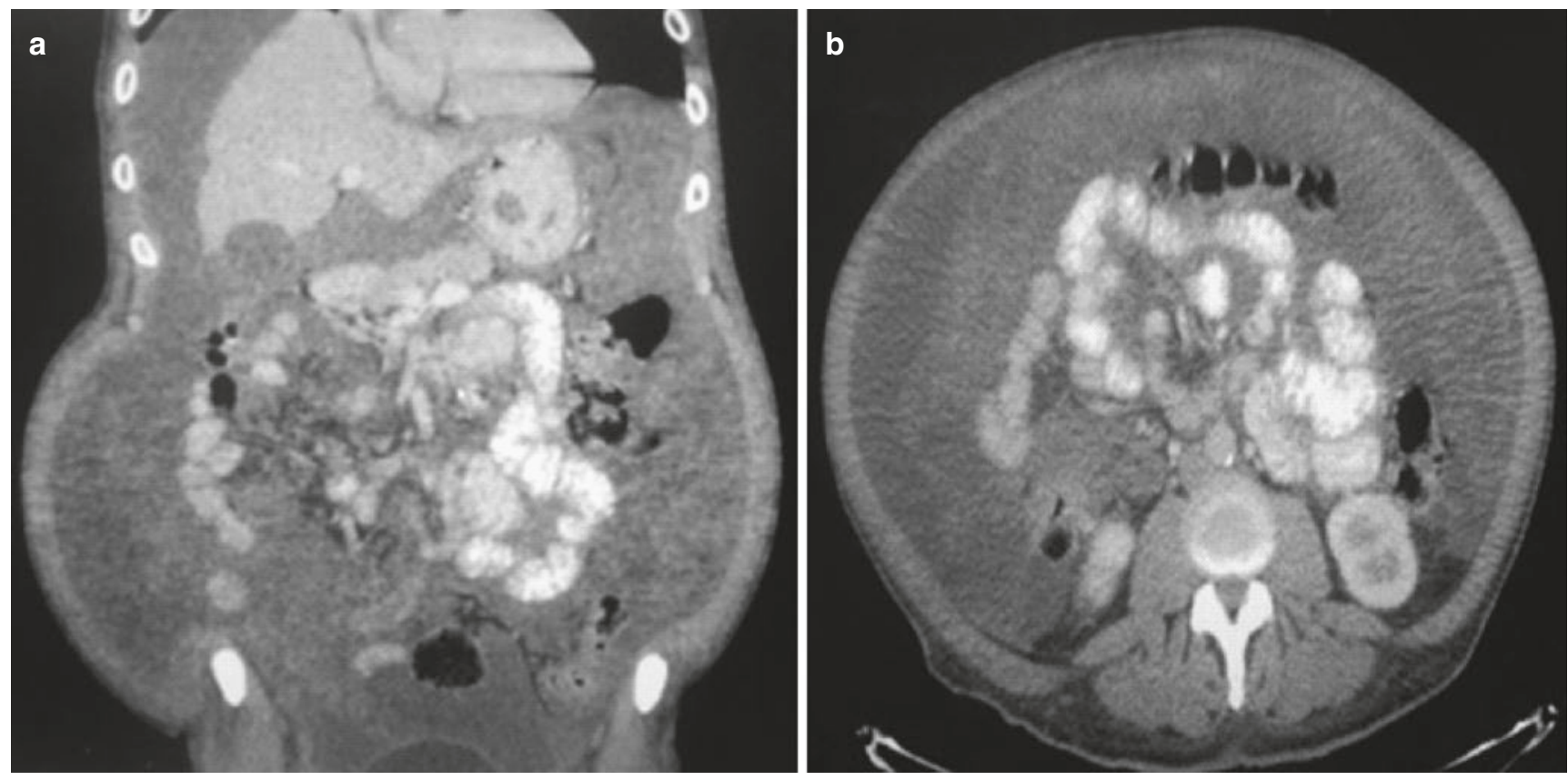

Fig. 23.3 (a, b) A patient's CT scan that shows a large volume of mucinous ascites and significant disease

Additional findings present on $\mathrm{CT}$ include ascites. Ascites of greater than $50 \mathrm{~mL}$ (and therefore identifiable on CT) is present in more than $70 \%$ of patients [2]. Ascites may be located freely throughout the abdomen or entrapped in different quadrants. Invasive peritoneal nodules can sometimes cause encasement of the large and small bowel on the serosa or mesentery resulting in complete or partial obstruction. Mucinous ascites will irritate the peritoneum causing a fibrotic reaction, which results in thickening of the peritoneal surfaces and may possibly lead to intestinal obstruction [2].

If not already surgically removed, the primary site of the cancer can also be sometimes identified-although a combination of endoscopy and laparoscopy may be necessary. It can be evaluated along with peritoneal spread and intraabdominal metastases. If no primary lesion is identifiable with imaging, endoscopy, and laparoscopy, a primary peritoneal neoplasm can be considered. Localized PC will be in close proximity to the primary lesion while diffuse is spread to most of the peritoneal surfaces. CT is particularly helpful when the head of the pancreas, porta hepatis, liver, and root of the mesentery are involved with metastatic disease [2]. The accuracy of CT decreases with assessment of small bowel disease and lesions $<5 \mathrm{~mm}$ [2].

MRI has demonstrated no diagnostic advantage over CT or in prediction of completeness of cytoreduction [2]. However, we recently implemented an MRI with peritoneal protocol that may have advantages in imaging the peritoneum, especially in the surveillance plan of young patients after surgery. Evaluation by PET scan (when used alone), often underestimates disease and may underestimate the disease burden when lesions are $<5 \mathrm{~mm}[11,12]$.

Furthermore, the ability of the radiologist to detect peritoneal disease on any imaging study may vary significantly between institutions, thus allowing centers with more experience to accumulate expertise through experience and familiarity with this patient group.

\section{Multidisciplinary Tumor Board}

The use of a multidisciplinary tumor board (MDT) provides a comprehensive approach to the patient's complex disease and extensive treatment history. It allows experts from a wide variety of medical and surgical specialties to prospectively review the patient's case and collectively determine the treatment most beneficial for the patient. The use of a multidisciplinary tumor board is now the standard in cancer care. The American College of Surgeons Commission on Cancer Program requires that each institution employ an MDT for case review and treatment decisions to receive proper accreditation [13]. An MDT is an education resource for providers, residents, and medical student; it increases awareness of different specialist's perspectives on the approach to specific cancers [13].

Each patient who presents with PC from a disseminated GI or gynecologic malignancy should be presented at the MDT. The internal structure of each meeting is variable according to institution. Typically, the patient's case is pre- 
Fig. 23.4 The peritoneal cancer index. (Reprinted with permission from Harmon and Sugarbaker [11]. Under the terms of the Creative Commons Attribution License (http://creativecommons.org/ licenses/by/2.0))

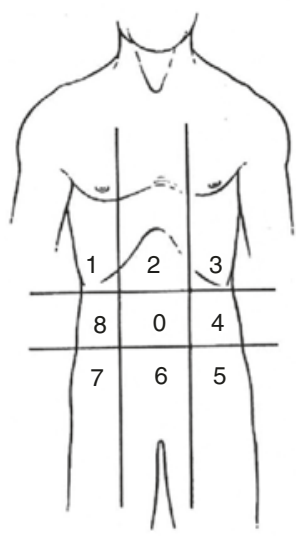

\section{Regions}

0 Central

1 Right Upper

2 Epigastrium

3 Left Upper

4 Left Flank

5 Left Lower

6 Pelvis

7 Right Lower

8 Right Flank

9 Upper Jejunum

10 Lower Jejunum

11 Upper lleum

12 Lower lleum

PCI

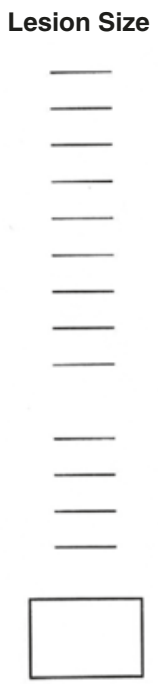

Lesion Size Score

LS 0 No tumor seen

LS 1 Tumor up to $0.5 \mathrm{~cm}$

LS 2 Tumor up to $5.0 \mathrm{~cm}$

LS 3 Tumor $>5.0 \mathrm{~cm}$ or confluence

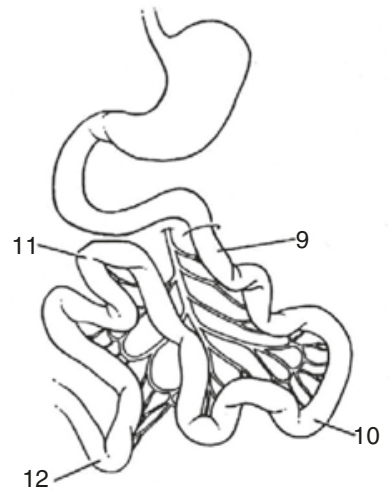

sented in detail: previous diagnosis, previous treatments (surgical and nonsurgical), reason(s) for presentation at the MDT, current physical exam, performance status, radiographic evidence, pathologic evidence, histology, and other elements of the patient's case are reviewed and discussed. Thus, all participants can contribute to developing a patientspecific treatment plan that could include further diagnostic tests, chemotherapy, radiation therapy, surgery, no intervention, or any combination thereof. It is essential that all cases of peritoneal cancer are routinely presented in GI tumors board meetings before treatment decision.

\section{Resection Guidelines/Operative Indications}

In the selection of operative candidates, one may consider the following: age, comorbidities, previous surgeries, previous chemotherapy/radiation, disease-free interval from previous interventions, histology of primary tumor, peritoneal cancer index (PCI), completeness of cytoreductive index (CCR) predicted and the peritoneal surface disease severity score (PSDSS), prior surgery score (PSS), and simplified PCI.

PCI is a tool used to quantify the disease in the abdomen and has been found to be an accurate assessment of survival [11]. It is most accurate when calculated at the time of operation, however preoperative radiographic evaluation shows reasonable sensitivity with high-volume tumors (100\%) [2]. However, radiographic PCI decreased in accuracy with small bowel involvement (sensitivity of $8-17 \%$ ) or lesions $<5 \mathrm{~mm}$ (sensitivity $11 \%$ ) [2]. It is a scoring system that divides the abdomen into 13 regions and a lesion size score (LSS) is assigned to each region [11]. Primary lesions or localized recurrences are excluded from the lesion size assessment (Fig. 23.4) [11]. The lesion sizes for all regions are summated and the total score is assigned from 0 to 39 .

Anatomic landmarks help divide the regions of the abdomen. The upper transverse plane is located at the lowest part of the costal margin; the lower transverse plane is located at the anterior superior iliac spine (ASIS). The sagittal planes divide into three equal columns. Region 0 is located at the umbilicus, region 1 is located in the right hemidiaphragm, and numbers continue in a clockwise direction [12]. Anatomic structures within each region are defined in Table 23.3 [11].

In particular, patients with colorectal cancer and other invasive cancers should have a thorough evaluation and a documented PCI before committing them to CRS and HIPEC. In 2010, a multicenter French study reported by Elias et al. looked at the role of the PCI in patients with colorectal carcinomatosis treated with surgery and HIPEC [14]. In this study it was shown that as the PCI increased above 20 in patients with CRC and PC, the survival dramatically declined despite having undergone CRS and HIPEC at an experienced center. In fact, in this data, there were no 5 -year survivors if the PCI was greater than 20, yet in those patients with a PCI of 1-6, the 5-year survival was over $40 \%$ with a median survival of 40 months [14]. At out our center we most often decline patients with PC from CRC and other invasive malignancies for HIPEC if the PCI is greater than 20. However, even if patients with invasive cancer have a PCI less than 20 that is not an absolute indication to proceed 
Table 23.3 Anatomic structures located in each abdominal region

\begin{tabular}{|c|c|}
\hline Regions & Anatomic structures \\
\hline 0 central & $\begin{array}{l}\text { Midline abdominal structures: greater omentum, } \\
\text { transverse colon }\end{array}$ \\
\hline 1 right upper & $\begin{array}{l}\text { Superior surface of the right lobe of the liver, } \\
\text { undersurface of the right hemidiaphragm, right } \\
\text { retro hepatic space }\end{array}$ \\
\hline 2 epigastrium & $\begin{array}{l}\text { Epigastric fat pad, left lobe of liver, lesser } \\
\text { omentum, falciform ligament }\end{array}$ \\
\hline 3 left upper & $\begin{array}{l}\text { Undersurface of the left hemidiaphragm, spleen, } \\
\text { tail of pancreas, anterior and posterior surfaces } \\
\text { of the stomach }\end{array}$ \\
\hline 4 left flank & Descending colon, left abdominal gutter \\
\hline 5 left lower & $\begin{array}{l}\text { Pelvic sidewall lateral to the sigmoid colon, } \\
\text { sigmoid colon }\end{array}$ \\
\hline 6 pelvis & $\begin{array}{l}\text { Female internal genitalia with ovaries, tubes and } \\
\text { uterus, bladder, pouch of Douglas, rectosigmoid } \\
\text { colon }\end{array}$ \\
\hline 7 right lower & Right pelvic side wall, cecum, appendix \\
\hline 8 right flank & Right abdominal gutter, ascending colon \\
\hline $\begin{array}{l}9 \text { upper jejunum } \\
10 \text { lower } \\
\text { jejunum } \\
11 \text { upper ileum } \\
12 \text { lower ileum }\end{array}$ & \\
\hline
\end{tabular}

Adapted from Harmon and Sugarbaker [11]. Under the terms of the Creative Commons Attribution License (http://creativecommons.org/ licenses/by/2.0)

with CRS and HIPEC. An example of this is in patients with an unresectable volume of tumor localized in one area, such as the porta hepatis; although the PCI is low, complete cytoreduction may not be possible and these patients generally should not be considered for HIPEC.

A noted exception to the PCI $>20$ rule is in those patients with low-grade appendiceal mucinous neoplasms (classic pseudomyxoma peritonei) or peritoneal mesothelioma. In those patients a PCI greater than 20 and even close to 39 can be found yet should not serve as a contraindication to cytoreduction and HIPEC because of the favorable long-term prognosis if those patients receive complete cytoreduction and HIPEC.

Arguably, the most important prognostic factor of treatment success is the CCR $[2,11,12]$. Multiple studies have shown improved survival in patients who had undergone complete cytoreduction for appendiceal, colorectal, and gastric cancers [11]. It is a good prognostic indicator for many different histopathologies. We urge that all patients who undergo cytoreduction should have the benefit of a team that can and always aims to achieve a complete cytoreduction. Complete cytoreduction versus incomplete cytoreduction are the main determinants. A CCR of 0 is achieved when there is no peritoneal seeding visualized within the abdomen after cytoreduction [11]. CCR 1 occurs when nodules $<2.5 \mathrm{~mm}$ persist after CRS [11]. A CCR 2 has residual nodules between $2.5 \mathrm{~mm}$ and $2.5 \mathrm{~cm}$, and a CCR 3 indicates nodules $>2.5 \mathrm{~cm}$
Table 23.4 Peritoneal surface disease severity score

\begin{tabular}{l|l|l}
\hline Clinical & PCI & Histology [colonic/appendiceal] \\
\hline $\begin{array}{l}\text { No } \\
\text { symptoms }\end{array}$ & $\begin{array}{l}\text { PCI } \\
<10 \\
\mathbf{1} \text { point }\end{array}$ & $\begin{array}{l}\text { Well differentiated } \\
\text { Moderately differentiated/N0 } \\
\text { Low-grade mucinous neoplasm } \\
\mathbf{1} \text { point }\end{array}$ \\
\hline $\begin{array}{l}\text { Mild } \\
\text { symptoms } \\
1 \text { point }\end{array}$ & $\begin{array}{l}\text { PCI } \\
10-20\end{array}$ & $\begin{array}{l}\text { Moderately differentiated/N1 or N2 } \\
\text { Mucinous adenocarcinoma } \\
\mathbf{3} \text { points }\end{array}$ \\
\hline $\begin{array}{l}\text { Severe } \\
\text { symptoms } \\
\text { 6 points }\end{array}$ & $\begin{array}{l}\text { PCI } \\
>20\end{array}$ & $\begin{array}{l}\text { Every poorly differentiated } \\
\text { Every signet ring } \\
\text { High-grade mixed adenocarcinoma and } \\
\text { goblet cell carcinoma } \\
\text { 9 points }\end{array}$ \\
\hline
\end{tabular}

Modified from Pelz et al. [12]

[11]. In order to proceed with HIPEC we advise a CCR of 0 or 1. This is due to the finding that the CCR-1 tumor size (but not CCR-2 and above) is thought to be penetrable by intraperitoneal chemotherapy, thus allowing HIPEC to complete the surgical cytoreduction. Furthermore, Sugarbaker and others have shown that the chance of survival for patients with a CCR 0 from a CRC with PC who underwent HIPEC to be $40 \%$ at 5 years, yet $0 \%$ survived 5 years when cytoreduction was incomplete (CCR 2 and above) [15].

In experienced centers, for very select patients with incomplete cytoreduction, such as those with refractory malignant ascites, HIPEC may be acceptable even though complete cytoreduction has not been achieved.

The peritoneal surface disease severity score (PSDSS) is another useful tool that incorporates clinical symptoms, extent of carcinomatosis, radiographic PCI, and tumor histopathology (Table 23.4) $[12,16]$. Although the initial use was for colon cancer with PC, its usefulness has been demonstrated in appendiceal cancer with PC, and our group has published an initial analysis on the usefulness of the stratifying system in our patients [12]. Mild symptoms are defined as weight loss of $<10 \%$ body weight, mild abdominal pain, and asymptomatic ascites [16]. Severe symptoms are defined as weight loss $>10 \%$ of body weight, refractory pain, bowel obstruction or symptomatic ascites [16]. A total score is calculated and correlated to a stage. A score of 2-3 points correlates to stage I disease; $4-7$ points correlate to stage II disease; $8-10$ points correlate to stage IV disease; and greater than 10 points correlate to stage IV disease. Patients who are stage I and II may benefit from cytoreduction and HIPEC, while stage III and IV rarely benefit from surgery.

In our institution, especially for CRC, the PSDSS is discussed preoperatively in the Tumor Board and it is shown to the patient. If the PSDSS is stage I, then we offer CRS and HIPEC upfront. In most cases if the PSDSS is stage II or above, we favor 3-6 cycles of systemic chemotherapy followed by restaging. In those who were stage II and who did not progress on systemic therapy, we then offer CRS and HIPEC. For 
those stage III on presentation, if after systemic therapy their performance status allows and they did not progress, then they may be offered surgery. Stage IV PSDSS patients on presentation rarely will become operative candidates and are not considered for HIPEC.

Our institution partnered with multiple other centers to publish data examining the role of PSDSS for patients with PC from CRC. The data was very encouraging for those patients with a low PSDSS stage who underwent CRS and HIPEC. In this study 78 patients with a PSDSS stage I had a median survival of 81 months and those with PSDSS stage II $(n=302)$ had a median survival of 49 months [17]. Although the intent of the study was not to look at survival and the patient groups were quite heterogeneous, the data appears quite encouraging. As may have been expected, those in the study with a PSDSS stage IV $(n=151)$ had a median survival of only 27 months. Thus, in most cases, this survival of stage III and IV patients, which may be equivalent to those who underwent systemic therapy alone, is not long enough to advocate for CRS and HIPEC in those patients with a high PSDSS [17].

\section{Prior Surgical Score}

The majority of patients with PC will have had some type of prior surgical intervention. This is extremely important as the extent of prior surgery before CRS and HIPEC can have a negative impact on survival and increase surgical morbidity. Sugarbaker has discussed the cancer entrapment phenomenon as surgical opening of tissue planes creating raw surfaces where cancer cells will adhere, become vascularized and progress. This can make subsequent surgical cytoreduction challenging or impossible, depending on the depth of penetration of the implanted cancer cells. A PSS of 0 means no prior surgery or biopsy only. A PSS of 1 indicates 1 region of surgery, PSS 2 indicates 2-5 regions, and PSS 3 indicates more than 5 regions previously explored and dissected.

While all of these scoring and stratifying systems alone may not be enough to fully evaluate and treat a patient with PC, the combined use of the ones discussed here may allow the treating team to help standardize their approach to these patients. Most importantly, they may allow patients who will benefit the opportunity for surgery, while sparing those who will not from unnecessary exploration.

\section{Operative Exploration/Technique}

Patients who are appropriate surgical candidates benefit from radical therapy and should be brought to the operating room with curative intent. Safety and optimal cytoreduction are the primary goals. A specialized team of surgeons, nurses, anesthesiologists, perfusionists, and pathologists must work together to create an optimal, safe, beneficial procedure.

Previously diagnosed comorbidities are present in $18 \%$ of patients undergoing major oncologic resection. These morbidities increase the risk of acute medical complications (OR 3.7), in-hospital mortality (OR 3.6), hospital costs, postoperative complications (OR 3.9), and increased complication severity (OR 3.6) [8]. It becomes imperative to have preoperative optimization of these morbidities. Particular attention should be paid to patients with previous cardiac medical history. The adverse effects and risk of general anesthetic effects on cardiac function are well documented. The anesthesiologist must also take into account the duration of the procedure and the effects of hypothermia and hyperthermia. The prolonged anesthetic exposure provides additional risk. Published studies demonstrate a range of operative times for CRS + HIPEC from 433 to 470 minutes [18-20]. Patients with previous coronary artery disease, congestive heart failure, or depressed left ventricular function may not be able to tolerate prolonged or aggressive intravenous fluid resuscitation [2]. Hyperthermia-induced increases in myocardial oxygen demand can cause devastating hemodynamic compromise [2]. The American Heart Association guidelines remain the accepted standard evaluation for preoperative cardiac assessment [2].

Special consideration in this patient population is the abdominal domain. Patients with PC can have large amounts of mucinous ascites (10-15 L) that have been collected within their abdomens [2]. The increased volume and pressure can result in a decreased functional residual capacity [2]. This is a risk factor for rapid oxygen desaturation, aspiration, and prolonged ventilator requirement [2].

Patient positioning is of vital importance. The prolonged operative time increases the risk of pressure-induced wounds. All pressure points should be supported and documented as such. Additional preventive measures such as the application of sequential compression devices (SCD) should be employed. The patient may be placed supine, flat, or lithotomy; we prefer lithotomy in anticipation of low anterior resection reconstruction. Upper extremities can be outstretched or tucked as long as no traction is placed on the extremity.

\section{Anesthesia}

A close relationship with the anesthesia team is essential for the success of the patient who undergoes CRS and HIPEC. Furthermore, the experience of the anesthesia team can help improve quality and outcomes. Hemodynamic monitoring is essential for intraoperative monitoring and patient safety. We advocate the use of arterial line for accu- 
rate blood pressure monitoring and selective use of central line placement for rapid administration of fluid, blood products, or inotropic support, if necessary. Some institutions do not routinely advocate the use of central line placement and we make this decision on a case-to-case basis. Although measurement of central venous pressure (CVP) does not accurately reflect volume status [2], central venous access improves the ease of serial lab draws: complete blood count, basic metabolic profile/complete metabolic profile, and coagulation studies. Close monitoring of such values allows prompt intervention and resuscitation if needed. Central lines expose the patient to potential risk: mechanical injury (pneumothorax, hematoma, bleeding, foreign body retention) and infection [2]. Other methods of estimating volume responsiveness include beat-to-beat changes in stroke volume (SV) induced by positive pressure ventilation (PPV) [2]. A small SV variation (12-13\%) can indicate volume responsiveness or euvolemia [2]. We do not routinely use a pulmonary artery catheter (Swan Ganz), however, we have employed its use in thoracoabdominal HIPEC cases and when a patient has a marked preoperative cardiac history. Most recently we have employed the use of noninvasive hemodynamic monitoring systems, mainly esophageal Doppler monitoring, which has been selectively studied and used in the management of patients undergoing HIPEC. Our initial results show benefit in terms of limiting volume replacement during the intraoperative course and we plan to analyze this more closely.

Additional dynamic monitoring methods that should be employed include esophageal temperature, bladder temperature, urine output, and close attention to ventilator settings during the operation (peak airway pressure, oxygen requirements). Frequent monitoring of these parameters, in addition to those listed previously, provide dynamic information that allows the surgeon and specialized team to monitor patient safety and intervene promptly when necessary.

During HIPEC, a hyperdynamic, vasodilatory state is induced [2]. The maximum change(s) are seen from 70 to 80 minutes (of the 90-minute intraperitoneal chemotherapy) [2]. Hemodynamic changes are induced by thermal stress; this translates into increased cardiac output, decreased systemic vascular resistance, increased heart rate, and increased end-tidal carbon dioxide [2]. The increase in cardiac output is primarily driven by an increase in heart rate and not myocardial oxygen demand [2]. Esquivel et al. observed these changes with intraoperative esophageal Doppler while using the "open coliseum" operative technique [21]. Consensus guidelines from the American Society of Peritoneal Surface Malignancies now advocate for the closed abdominal technique [22]. This technique can increase intra-abdominal pressure and further exaggerate hemodynamic changes.

One of the biggest intraoperative challenges for the surgeon and anesthesiology team is fluid management. It becomes tempting to administer large amounts of crystalloid fluid in response to changes in the central venous pressures (CVP) or "third space" losses. This method can result in increased postoperative complications: pulmonary edema, adult respiratory distress syndrome (ARDS), impaired healing of anastomoses, and dilution of coagulation factors and platelets, which could result in a clinically significant coagulopathy [2]. There is good documentation that the judicious use of intraoperative fluid(s) improves outcomes after major gastrointestinal surgery [23]. Frequent laboratory draws, urine output, heart rate, ventilator parameters, stroke volume, cardiac output, and other means can all be used to provide a reasonable picture of patient status during the CRS and HIPEC. Targeted resuscitation should be emphasized with use of crystalloid fluid, synthetic colloid, and humanderived colloid (blood products). Average estimated blood loss (EBL) at our institution is approximately $240 \mathrm{~mL}$, with literature citing an average EBL 300-500 mL [2]. However, with any large hemorrhage ( $>500 \mathrm{~mL})$, volume replacement with blood products should be discussed. Additional consideration for this patient population is decreased oncotic pressure that may occur secondary to a loss in protein from surgically removed ascites. The use of synthetic colloid as replacement may need to be considered. The restrictive use of fluids during HIPEC has recently translated into improved postoperative outcomes, however, it should be stressed that adequate resuscitation and renal perfusion must also be achieved during the perfusion.

Accurate and frequent measurement of urine output is essential for hemodynamic status and renal preservation; some chemotherapeutic agents are known nephrotoxins. Ensuring adequate intravascular volume is essential as increased cardiac output and decreased vascular resistance can increase renal blood flow and renal perfusion during HIPEC [2, 24]. Dopamine was once thought to provide a nephron-protective effect by stimulation of the DA1 receptors (renal vasodilation and inhibition of proximal tubule active sodium transport) [24]. However, this is now less accepted and administration of dopamine during HIPEC is not advocated by the consensus guidelines [22]. However, we use low-dose dopamine during the perfusion in the majority of cases, but acknowledge the potential lack of benefit. If increased diuresis is needed despite adequate intravascular volume and renal perfusion, the administration of furosemide can provide additional diuresis [2]. Furthermore, the use of vasopressors to increase renal perfusion has been used during the perfusion in our and other series with acceptable return of increased urine output.

In addition to ensuring adequate volume status, electrolyte disturbances (such as potassium) must be corrected prior to administration of chemotherapeutic agents to prevent exaggerated effects due to renal losses. Certain chemotherapeutic agents can cause electrolyte disturbances. In rare 
instances, oxaliplatin can predispose to a lactic acidosis, hyperglycemia, and hyponatremia [2]. Cisplatin can cause cardiac dysrhythmias (specifically ventricular tachycardia) by altered magnesium levels [25]. Additional observations comparing mitomycin $\mathrm{C}$ to oxaliplatin showed that patients receiving oxaliplatin had significant 24-hour postoperative hyponatremia, hyperglycemia, and metabolic acidosis, and thus strict intraoperative glycemic control is essential [26].

Furthermore, at our institution over a 24-month period we have implemented a system to monitor intra-abdominal pressure during the HIPEC. This is intended to help allow for additional data to manage the patient's volume status, hemodynamic changes, and urine output changes during the perfusion. For example, we have experienced in some cases a rapid change or decline in urine output when intra-abdominal pressures exceed $22 \mathrm{~mm} \mathrm{Hg}$ and thus this monitoring allows us to adjust the pressure by changing the volume of the perfusate. Importantly, the pressure monitoring also allows us to maintain an adequate pressure that helps ensure optimal penetration of the chemotherapeutic agent being used. This work will be published in an upcoming review by the authors.

\section{Diagnostic Laparoscopy}

Traditionally, laparoscopic staging was discouraged due to difficulty in trocar placement in the presence of abdominal wall mass(es) or multiple previous surgeries, neoplastic contamination of port sites, and skepticism about the reliability of the procedure [10]. However, this is being challenged and more institutions, including ours, are performing diagnostic laparoscopy (DL) regularly. We use an open Hasson technique for laparoscopy and favor the left upper quadrant if feasible (Fig. 23.5). DL allows the surgeon to calculate the extent of disease and assess tumor burden, and determine the PCI and extent of resection needed to achieve CCR or $0 / 1$ with less operative time, less morbidity and mortality compared to a laparotomy. DL has demonstrated multiple strengths: evaluation of small bowel mesentery, through evaluation of all regions of the PCI scoring regions; evaluation of the omental bursa, pelvic cavity, diaphragm, and abdominal wall; and allow for peritoneal washings and biopsies if needed to determine the course of treatment [10]. Its areas of inherent weakness pertain to evaluation of the thickness of diaphragmatic lesions and evaluation of pancreatic or lesser sac involvement; however, with the use of intraoperative laparoscopic ultrasound these challenges could be overcome [10].

Indications for DL include staging of PC already diagnosed via imaging, staging of PC of unknown origin, restaging following neoadjuvant therapy, restaging during follow-up with uncertain imaging, and restaging following adjuvant therapy [10].

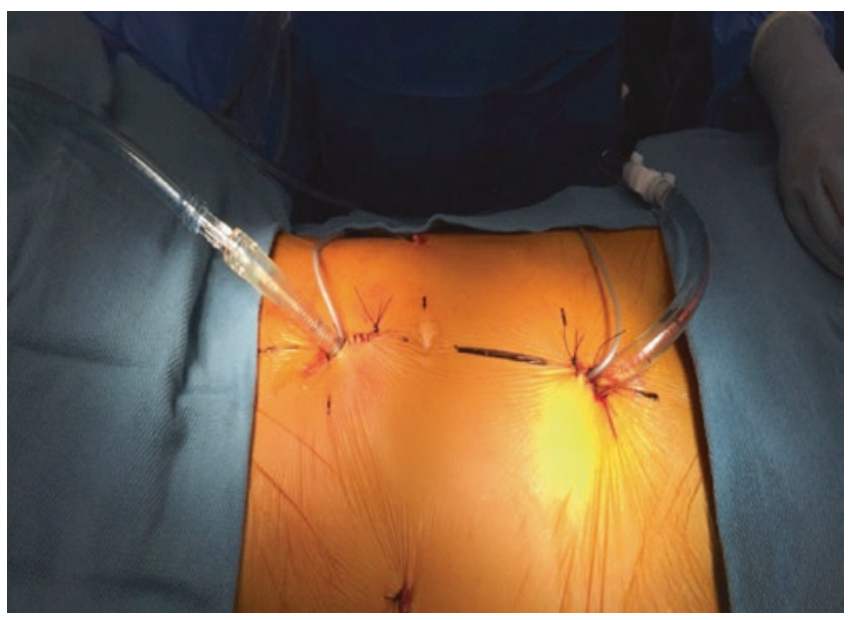

Fig. 23.5 Laparoscopic patient: our four-trocar approach to laparoscopic HIPEC in a patient with very low volume, low-grade appendiceal mucinous disease. This patient underwent laparoscopic HIPEC (shown here) and was discharged on POD 2

At our institution a patient may be brought for DL 2 weeks prior to a potential CRS + HIPEC or immediately prior to CRS + HIPEC. If a patient is brought to the operating room for diagnostic laparoscopy 2 weeks prior to potential CRS + HIPEC, he or she can be admitted as "same-day surgery" with admission and discharge the same day. Bowel preparation is usually not required. The patient is placed under general anesthetic with endotracheal intubation. Typically, patients previously have had (multiple) gastrointestinal surgical procedures; therefore using the Hasson technique to enter the abdomen is most safe in our experience. Location of entry is based on surgeon preference: periumbilical, right or left flank, right or left iliac fossa, midaxillary line, or left upper quadrant (our preferred site of entry) [10]. In our approach, once the fascia is grasped and incised, great care should be taken to dissect away any adhesions avoiding bowel injury; and once deemed safe, a largediameter blunt Hasson port (10-12 mm) should be inserted gently and secured. Ascites should be evacuated prior to pneumoperitoneum being established [10]. If the patient has a large amount of ascites, pneumoperitoneum may be difficult to obtain without high intra-abdominal pressures. Some of these patients will be able to tolerate higher pressures due to chronic domain expansion due to ascites; however, the surgeon must be astute to subtle hemodynamic changes to indicate hemodynamic compromise and stop the procedure.

When diagnostic laparoscopy is performed immediately preceding CRS + HIPEC very little differences exist. Patients should be prepared and admitted with expectation for CRS + HIPEC to be completed. Prior to surgery, patients should have completed a bowel preparation, recent imaging (CT scan within the last 1-3 months), full laboratory work 
(CBC, BMP, INR, etc.), electrocardiogram (EKG), and any other preoperative testing needed to optimize the patient.

Upon visualization of the abdomen, each quadrant of the abdomen and the entire peritoneum should be visualized if possible and a PCI calculated. The patient should be rotated into at least four different positions to fully inspect the abdomen: steep reverse Trendelenburg left tilt, steep reverse Trendelenburg right tilt, steep Trendelenburg right tilt, and steep Trendelenburg left tilt [10].

In one report, diagnostic laparoscopy was performed in 351 patients with $99.7 \%$ of patients having successful staging [10]. Only 1 patient $(0.28 \%)$ was not able to undergo laparoscopy staging due to dense adhesions [2]. Five patients were under staged (1.4\%) which became evident upon laparotomy and resulted in incomplete cytoreduction [10]. There were two site infections, one episode of bleeding, one bowel perforation, one diaphragm perforation, and zero mortality [10]. No neoplastic seeding was detected or any port site metastases [10].

The algorithm for proceeding with CRS + HIPEC after DL is outlined in Fig. 23.6 [10]. This algorithm is based on a combination of absolute exclusion criteria and relative inclusion criteria if a patient has an acceptable PCI on DL.

If the patient is not a candidate for CRS + HIPEC, the patient can be discharged that day with short-term follow-up in the office to discuss surgical findings and referral to medical oncol- ogist. If the patient is eligible for and able to complete further systemic treatments, they can then at that point be brought back to the surgeon's office for re-evaluation and restaging. We prefer performing diagnostic laparoscopy in a separate setting before the intended CRS and HIPEC, especially for high-grade histologies. This helps limit the mobilization of extensive resources for the major procedure when the diagnostic laparoscopy reveals an unresectable burden of disease.

\section{Cytoreductive Surgery}

If a patient is deemed an acceptable candidate for CRS + HIPEC after laparoscopic evaluation, the pneumoperitoneum is evacuated, trocars removed, and laparotomy performed via midline incision. In some patients with very limited disease (PCI $<10)$, laparoscopic cytoreductive surgery may be possible $[27,28]$. Esquivel et al. demonstrated that in patients with limited disease, laparoscopic cytoreduction is feasible and safe [27]. A European study showed that when comparing laparoscopic versus open procedures, compete cytoreduction was possible without conversion to open [28]. The laparoscopic group had a shorter mean operative time (210 versus 240 minutes), shorter mean length of stay (12 versus 19 days), and fewer grade III/IV complications (one versus four) [28].
Fig. 23.6 As from Valle et al. [10], algorithm for decisionmaking process for a correct indication for radical CRS + HIPEC based on laparoscopic staging
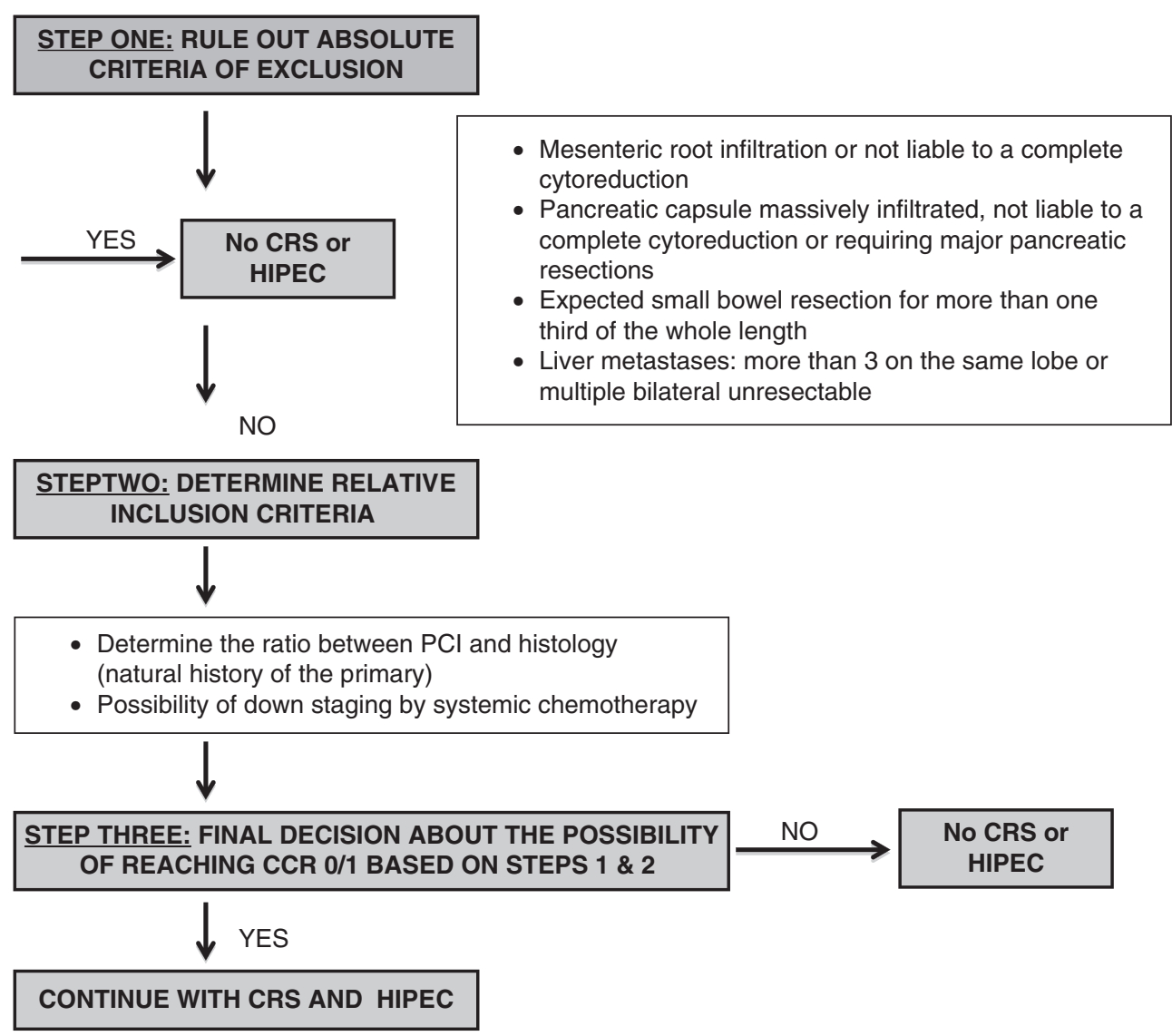
To proceed with laparotomy for CRS, a midline incision is extended superiorly to the xiphoid (which we routinely resect) and inferiorly elliptically around the umbilicus (which is resected) to the pubis. Great care must be taken when entering the abdomen to prevent inadvertent organ injury. Upon entrance into the abdomen, a thorough manual inspection should be performed. The surgeon should evaluate all regions: the retrohepatic space, the lesser sac, the splenorenal fossa, the pelvis, small bowel, the mesentery, and entire peritoneum. If there is an acceptable PCI $(<20)$ in patients with invasive disease, then cytoreductive surgery (CRS) with curative intent should proceed; this, of course, depends on the primary histology.

A large-caliber, self-retaining retractor that exposes the entire abdomen should be utilized. In our practice the Thompson liver retractor (Thompson Surgical Instruments, Inc., Traverse City, MI) is utilized to expose the entire abdomen. Surgeons should also take into consideration the tools that are used to resect tumor. Traditional scissor and knife resections can cause profuse bleeding from peritonectomy and cause a large dissemination of malignant cells within the abdomen [29]. The use of electrocautery/electroevaporative surgery should be implemented. A zone of heat necrosis (at the margin of resection) is caused by high-voltage electrocautery, which destroys all malignant cells within this zone [29].

Lysis of all adhesions should precede for all peritonectomies or visceral resections. It is theorized that malignant cells are trapped within adhesions, which are not penetrated by the chemoperfusate [29]. The "tumor cell entrapment hypothesis" is a mechanism whereby malignant cells are fixed at sites of prior surgical dissection [29]. It is therefore of vital importance to take down all adhesions and preserve bowel integrity as much as technically possible.

Cytoreductive surgery for peritoneal malignancies includes resection of primary tumor(s) and all metastases; this may include the entire peritoneum. Up to five procedures may be needed to achieve resection of the peritoneum that is involved with the malignancy [29]. The peritonectomy procedures include: anterior parietal, left upper quadrant, right upper quadrant, pelvic, and omental bursectomy [29]. Please see Table 23.5 for the resection regions achieved by each peritonectomy [29].

Both parietal and visceral peritoneum may need resection; however, when the visceral peritoneum is involved the underlying organ (stomach, small bowel) requires coinciding resection [29]. The visceral peritoneum is involved most commonly in three locations: the rectosigmoid colon, ileocecal valve, and antrum of the stomach [29]. These three locations are sites where the bowel and retroperitoneum have a particularly strong attachment with less peristalsis of the visceral peritoneum allowing more time for tumor deposition [29]. A complete pelvic peritonectomy is most often required:
Table 23.5 The five different peritonectomy procedures and their regions of resection for each

\begin{tabular}{l|l}
\hline $\begin{array}{l}\text { Peritonectomy } \\
\text { procedures }\end{array}$ & Resection regions \\
\hline Anterior parietal & $\begin{array}{l}\text { Epigastric fat pad, umbilicus, and previous } \\
\text { incisions of the abdomen }\end{array}$ \\
\hline Left upper quadrant & Greater omentum and spleen \\
\hline $\begin{array}{l}\text { Right upper } \\
\text { quadrant }\end{array}$ & Tumor on Glisson's capsule \\
\hline $\begin{array}{l}\text { Pelvic } \\
\text { peritonectomy }\end{array}$ & Uterus, ovaries and rectosigmoid junction \\
\hline $\begin{array}{l}\text { Omental } \\
\text { bursectomy }\end{array}$ & Gallbladder and lesser omentum \\
\hline
\end{tabular}

Modified from Sugarbaker [29]

stripping of all sidewalls, peritoneum overlying the bladder, the cul-de-sac, and resection of the rectosigmoid colon [29]. Resection of the ileocecal valve along with the distal most terminal ileum is often required [29]. The pylorus of the stomach is fixed to the retroperitoneum, and tumor may collect in the subpyloric space via the foramen of Winslow [29]. Large amounts of disease in this area may cause gastric outlet obstruction [29].

Multiple additional procedures may need to be performed to obtain CCR $0 / 1$. If a (right or left) subdiaphragmatic peritonectomy is to be performed, we advocate for xiphoidectomy prior to the peritonectomy [29]. The xiphoid is exposed back to its origin at the base of the sternum using electrocautery, which has twofold importance in this area: to control arterial bleeding that is located lateral to the xiphoid and to allow easier fracture of the xiphoid due to denatured bone proteins [29]. The xiphoid can be grasped with a Kocher clamp or similar tool and fractured away sharply from the base of the sternum.

Alternatively, we prefer that after dissection through the abdominal wall, prior to entrance into the abdomen, the surgeon dissect the parietal peritoneum off the retrorectus sheath. This leaves the anterior peritoneum intact and a small peritoneal window at the superior aspect of the incision can be created (Fig. 23.7). This will allow the surgeon to inspect and palpate the anterior parietal peritoneum and assess if a total or partial anterior parietal peritonectomy is needed [29]. Dissection should continue superiorly to the undersurface of the hemidiaphragm(s) down toward the paracolic gutters [10]. The section of the parietal peritoneum in closest attachment with the underlying tissue is along the transverses muscle. Dissection is more difficult here compared to the looser connections along lines of Toldt along the paracolic sulcus [10]. If cancer nodules are palpated, a complete anterior peritonectomy is required; if no nodules are palpated, then the anterior peritoneum can be maintained with only regional resections.

A left subphrenic peritonectomy is begun by dissection of the epigastric fat pad and peritoneum off the posterior rectus 


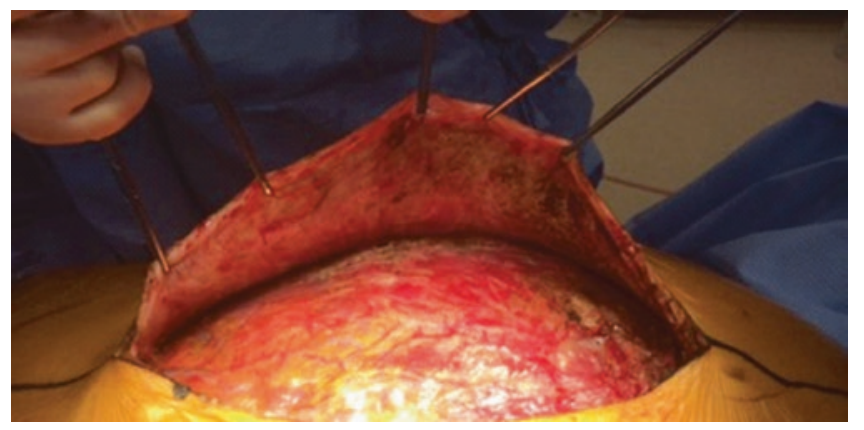

Fig. 23.7 Anterior parietal peritonectomy in our patient with a lowgrade appendiceal mucinous carcinomatosis with large volume mucinous ascites

sheath [29]. Dissection continues with electrocautery to separate the peritoneum from the diaphragm, left adrenal gland, and superior portion of the perinephric tissue [29]. The splenic flexure of the colon should be mobilized medially by transection of the peritoneum along the lines of Toldt [29]. The stomach (after ligation and transection of all of the short gastric arteries) can be reflected medially to allow visualization of the left adrenal gland, pancreas, the anterior surface of the transverse mesocolon, and perinephric tissues [29]. The left lateral liver should be mobilized, with care not to injure the inferior phrenic vein, which can be ligated and divided if needed to perform inclusive peritonectomy. At this point we also incise the pars flaccida to allow access to the lesser omentum and caudate liver, which should be explored thoroughly. Blood vessels that are encountered during dissection of the diaphragm should be well controlled prior to division, for these vessels tend to retract into the diaphragm muscle, which can be a source for ongoing hemorrhage [29].

A right subphrenic peritonectomy begins similar to that of the left: from the right posterior rectus sheath. Dissection should be continued in the same manner, using high voltage $3 \mathrm{~mm}$ ball tip electrocautery, taking care to control all vessels encountered. To ensure complete peritonectomy, mobilization of the liver must be extensive and gentle downward retraction should be used so as to not damage the liver or its vascular attachments. The right peritonectomy is continued until the bare area of the liver is reached [29]. The peritoneum should be followed onto the liver surface as Glisson's capsule. All of the capsule and associated tumor should be removed. It is possible to remove a thick layer of tumor with little blood loss by using electrocautery beneath Glisson's capsule [29]. Complete removal of the falciform ligament, most importantly at this area of hepatic attachment, is necessary. Not only is tumor deposition along the falciform ligament encountered, but at its entrance in the hepatic parenchyma it is covered in peritoneum, creating a tunnel with potential tumor deposition [29]. In some patients a bridge of hepatic tissue covers the entrance; this bridge must be divided to allow full inspection of this area of peritoneum
[29]. This is often in close proximity to the left hepatic artery, so careful, direct dissection must occur [29]. See Fig. 23.8 for right upper quadrant peritonectomy intraoperative and completion.

Lateral dissection over the perinephric tissues and right adrenal gland should also be completed [29]. If tumor is densely adherent to or invading the tendinous portion of the diaphragm, that section should be resected using an elliptical excision and promptly repaired with a strong nonabsorbable 0 suture [29].

Removal of the gallbladder should occur in standard fundus down technique. Once the cystic duct and cystic artery are ligated, the tumor overlying the hepatoduodenal ligament can be removed [29]. Oftentimes, tumor is heavily layered over the ligament, but this can be dissected away bluntly [29]. However, thick deposits of tumor can make cystic dissection difficult due to skewed anatomy.

We prefer to encircle the porta hepatis and then dissect out all structures as the tumor is dissected away. The lesser omentum is resected with preservation of the right gastric artery [29]. One must inspect for the presence of a replaced or accessory left hepatic artery coming from the left gastric artery. This must be preserved unless embedded in tumor and its preservation would prevent a complete cytoreduction [29]. The gastrohepatic ligament is separated from its hepatic attachments at segments 2 and 3, with careful dissection around the caudate lobe to not disrupt its delicate blood vessels, which has its origins along the anterior surface of those segments [29]. The peritoneum and lesser omentum is divided along the lesser curvature of the stomach [29]. It is separated from the vascular and vagal arcades toward the left gastric artery and subsequently released [29].

Reflection of the left liver can allow the surgeon to visualize the posterior aspect of the hepatoduodenal ligament and omental bursa. The peritoneum overlying the left liver extending to the subhepatic vena cava is divided. Blunt dissection can then be used to strip the peritoneum from the superior recess of the omental bursa, the crus of the right diaphragm, and beneath the portal vein [29].

A complete pelvic peritonectomy includes resection of the uterus, ovaries, rectosigmoid colon, and peritoneum [29]. Pelvic peritonectomy begins with resection of the peritoneum from the inferior aspect of the abdominal incision. Dissection is continued to the right and left borders of the bladder [29]. The peritoneum overlying the surface of the bladder is stripped away to the level of the cervix or seminal vesicles while counter traction is placed on the urachus [29]. The proper plane for dissection is between the musculature of the bladder and its overlying fatty tissue [29]. Both uterine arteries are ligated close to the base of the bladder, just above the ureters [29]. Laterally, the peritoneum is continuous with the peritoneum of the right and left 


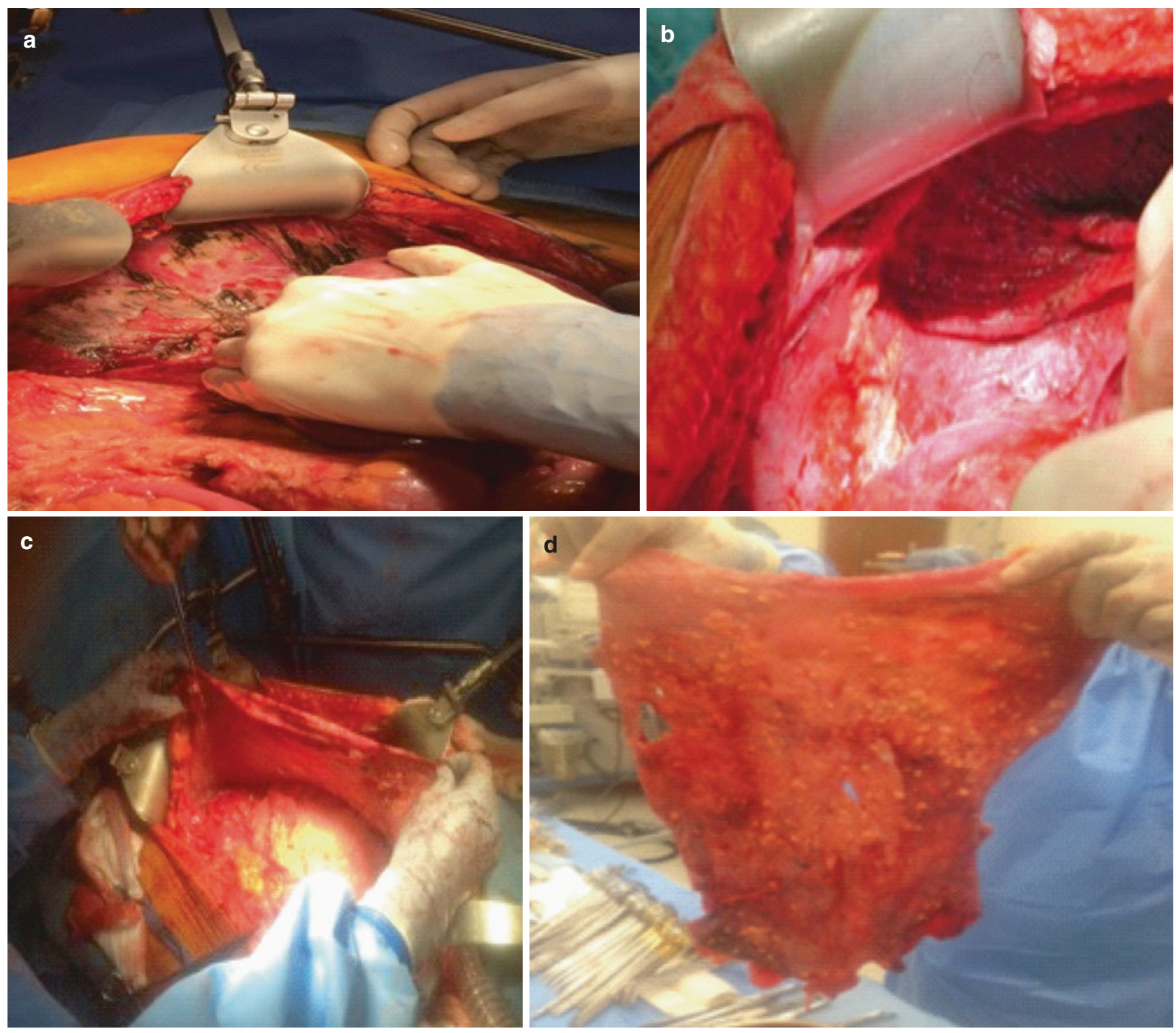

Fig. 23.8 (a, b) Retraction of the liver demonstrating the right upper quadrant peritonectomy site. (c) Left upper quadrant/abdominal wall peritoneum. (d) The right upper quadrant with liver retraction demonstrating a complete peritonectomy and the removed specimen

paracolic sulci [29]. Care must be taken not to damage the ureters. In females, the round ligament is identified and ligated as it enters the internal inguinal ring [29]. Both ovarian veins are ligated at the level of the lower pole of the kidney [29]. If tumor burden is present beyond local resection, the rectosigmoid colon is formally resected just distal to the pelvic tumor [29]. Electrocautery is used to excise the mesorectum circumferentially [29]. Exposure of the rectovaginal septum is then achieved by dissecting the bladder away from the cervix where the anterior and posterior vaginal cuff is transected [29]. The perirectal adipose is divided beneath the peritoneal reflection to ensure removal of all tumor within the cul-de-sac [29]. See Fig. 23.9 for pelvic peritonectomy.
Small bowel involvement may be extensive or focal. There are five types of small bowel involvement based on size and invasiveness. See Table 23.6 [30].

Type 1 nodules are small in size, do not invade past the peritoneum and have a less aggressive histology [29]. The small size of these nodules are amenable to resection using scissors and do not require resection of the small bowel wall [29]. Type 2 lesions require a partial thickness resection of the bowel wall due to invasion into the muscular layer [29]. Mucosa and submucosa are left intact and the seromuscular layer is repaired primarily [29]. These nodules are preferentially removed via scissor dissection. Type 3 nodules are large enough that a full-thickness resection of the antimesenteric bowel wall is needed [29]. The defect is 
repaired in a two-layered fashion. Type 4 nodules can undergo localized resection or segmental small bowel resection pending the size (of the nodule) and vascular sup-
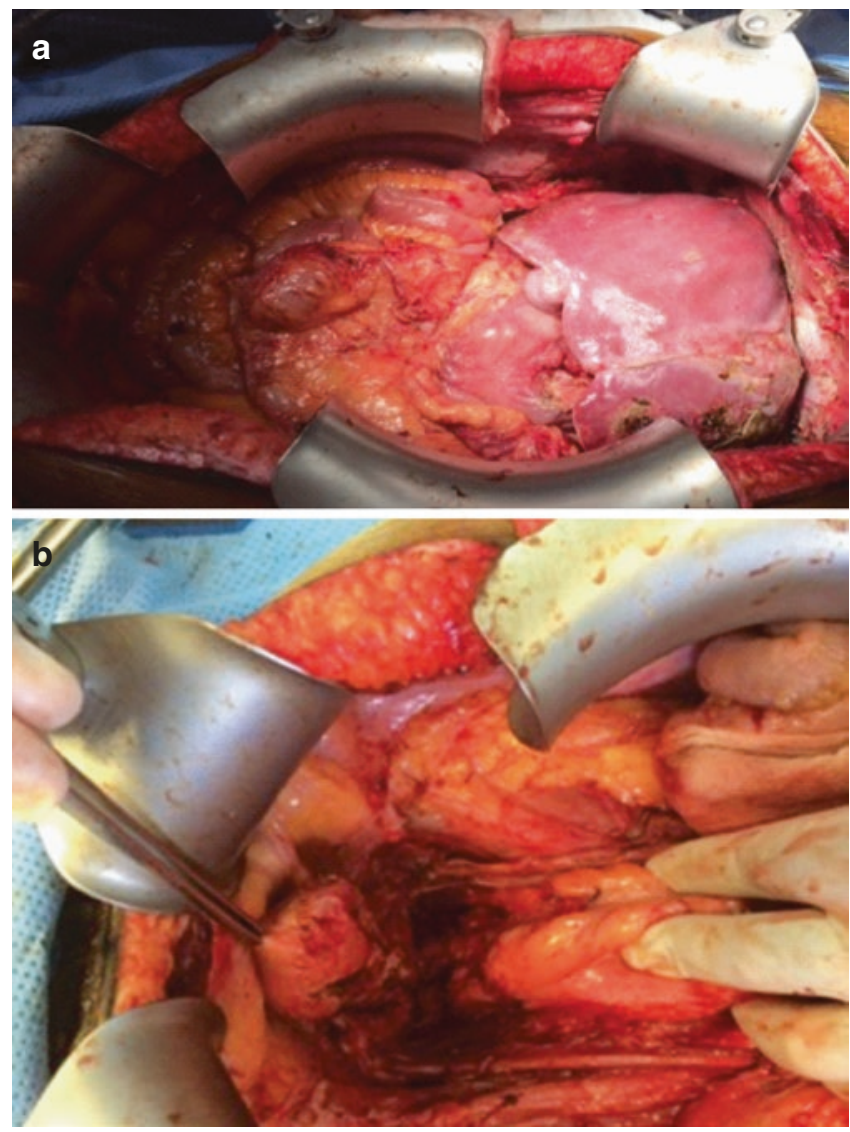

Fig. 23.9 (a, b) View into the pelvis demonstrating complete removal of pelvic peritonectomy and abdominal view after complete cytoreduction ply [29]. A two-layered repair follows the localized resection, and a hand sewn end-to-end or stapled side-to-side small bowel anastomoses are performed for segmental resection. Type 5 nodules require a formal small bowel resection with associated mesentery (Fig. 23.10) [29]. The section of small bowel and mesentery that is resected is divided with a linear stapler.

Currently there is no consensus if anastomoses should occur prior to or after chemoperfusion of the abdominal cavity. We routinely perform all anastomoses after perfusion. The only agreed upon closure prior to HIPEC is that of the vaginal cuff to prevent leakage. An observational study over a 10-year period demonstrated no difference in the development of digestive fistulas in patients who had anastomoses performed prior to (26\%) or after (74\%) HIPEC was performed [31]. Full bowel resections with primary anastomoses should be completed after HIPEC (i.e., type 5 small bowel nodules). In our center all bowel anastomoses are performed after HIPEC.

Table 23.6 The five types of small bowel involvement

\begin{tabular}{l|l}
\hline $\begin{array}{l}\text { Nodule } \\
\text { type }\end{array}$ & Description \\
\hline Type 1 & Noninvasive nodule \\
\hline Type 2 & $\begin{array}{l}\text { Small invasive nodules on the anti-mesenteric portion of } \\
\text { the small bowel }\end{array}$ \\
\hline Type 3 & $\begin{array}{l}\text { Moderate sized invasive nodules on the anti-mesenteric } \\
\text { portion of the small bowel }\end{array}$ \\
\hline Type 4 & $\begin{array}{l}\text { All sizes of invasive nodules at the junction of small } \\
\text { bowel and its mesentery }\end{array}$ \\
\hline Type 5 & Large invasive nodules \\
\hline
\end{tabular}

Modified with permission from Bijelic and Sugarbaker [30]
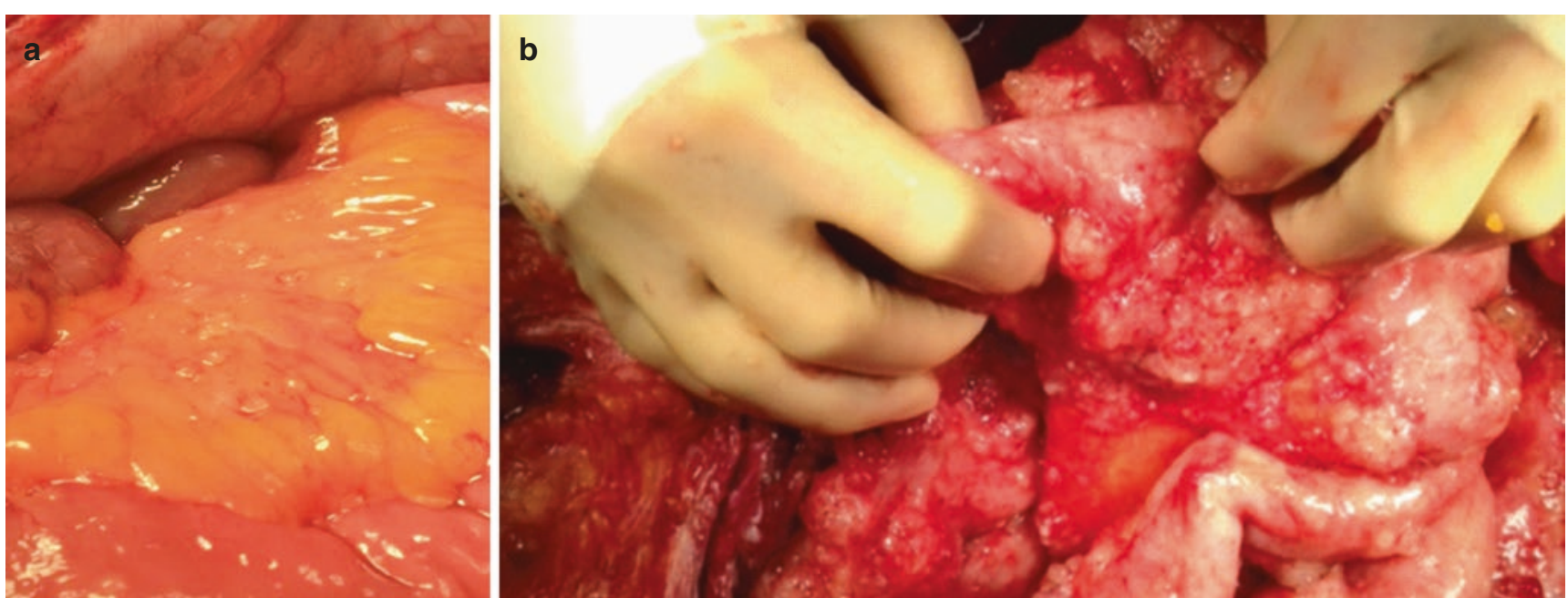

Fig. 23.10 (a) Small noninvasive resectable nodules in small bowel mesentery. (b) Invasive nodules of various sizes on the small bowel 


\section{Hyperthermic Intraperitoneal Chemotherapy (HIPEC)}

Once a CCR 0/1 has been achieved, the team can begin to prepare for instillation of the chemoperfusate. HIPEC should achieve total destruction of all microscopic in situ malignant cells. Animal models have supported a "peritoneum-tumor" barrier composed of the peritoneal mesothelium, the extracellular matrix that surrounds the tumor and successive layers of tumor cells [32]. This barrier is a limiting factor for penetration of the chemoperfusate into the tumor.

In previous literature, multiple different techniques to perform HIPEC have been described. Variability existed from institution to institution based on HIPEC method (open coliseum, partial closure, peritoneal cavity expander, closed), drug(s) used, dosage of drug(s), timing of drug delivery, volume of perfusate, inflow temperature, and duration of perfusion.

The American Society of Peritoneal Surface Malignancies (ASPSM) was created to develop standardized methods of patient selection and therapy guidelines to maximize benefit while minimizing morbidity and overtreatment of this diverse patient population [22]. As of 2017, the ASPSM had 240 members from 26 countries [33]. When established in 2009, the first goal of the ASPSM was to establish standardization of HIPEC delivery in the United States of America for multiple disease processes (colorectal cancer, ovarian cancer, peritoneal mesothelioma, low-grade appendiceal, and high-grade appendiceal cancers) $[22,33]$. To date, the consensus guidelines on HIPEC delivery for use in colorectal cancer with peritoneal dissemination have been published [22].

Traditionally, three methods of HIPEC delivery have been described: open coliseum technique, the peritoneal cavity expander (PCE), and closed technique [2]. Although most centers now perform exclusively closed technique, we will briefly mention the open coliseum and PCE techniques. The open method, often referred to as the coliseum technique, was originally described by Sugarbaker upon completion of CRS, four watertight closed outflow suction drains are anchored through the abdominal wall [2]. These drains remain in place in the postoperative period [2]. An inflow line is placed over the open abdomen into the peritoneal cavity along with accompanying temperature probes [2]. The abdominal incision skin edges are suspended to create a selfretaining column with the surgical retractor (Fig. 23.11) [34, 35]. A plastic sheet is placed over the abdominal opening that contains a small incision to allow the surgeons to manually stir the cavity [2]. Personal protection equipment is of vital importance to surgeon safety with this technique (double glove, goggles, imperforate gown, etc.) [2].

The peritoneal cavity expander is a variation of the open coliseum technique that was utilized in Japan without much popularity elsewhere $[2,36]$. This method utilizes an acrylic cylinder with inflow and outflow lines that are secured over the abdominal incision [2, 36, 37]. See Figure 23.12 [36]. When the expander is filled with perfusate, it allows the small bowel to float, allowing it to be manipulated [2].

The closed technique is the method most widely practiced and described in the ASPSM consensus guidelines. Once cytoreduction has been achieved, the abdomen is thoroughly irrigated to remove any cellular debris. Perfusion cannulas are attached to inflow catheters with a watertight 0 silk suture (or similar suture). Temperature and pressure probes are attached to the cannulas with a suture in a similar fashion. Inflow and outflow catheters are placed under the diaphragm and into the pelvis. The tubing must lie easily without kinks or sharp bends. The laparotomy incision is then closed, watertight, around the cannulas, creating a closed circuit.

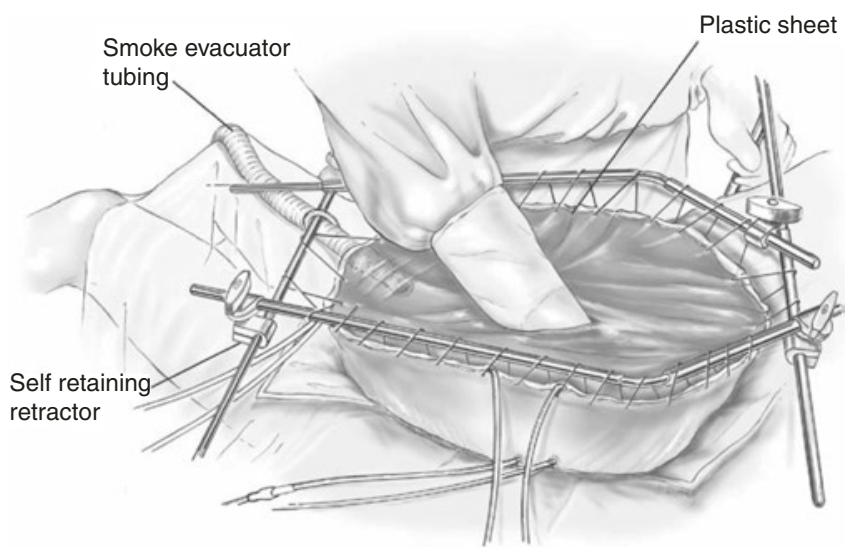

Fig. 23.11 Demonstration of the open coliseum HIPEC technique. (Reprinted with permission from Esquivel et al. [35])

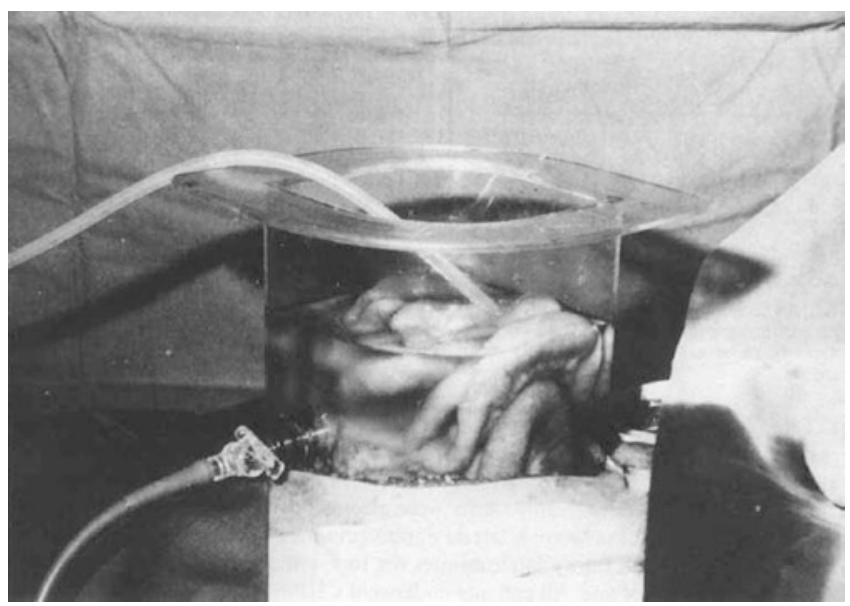

Fig. 23.12 Demonstration of the peritoneal cavity expander HIPEC technique. (Reprinted with permission from Fujimura et al. [36]) 

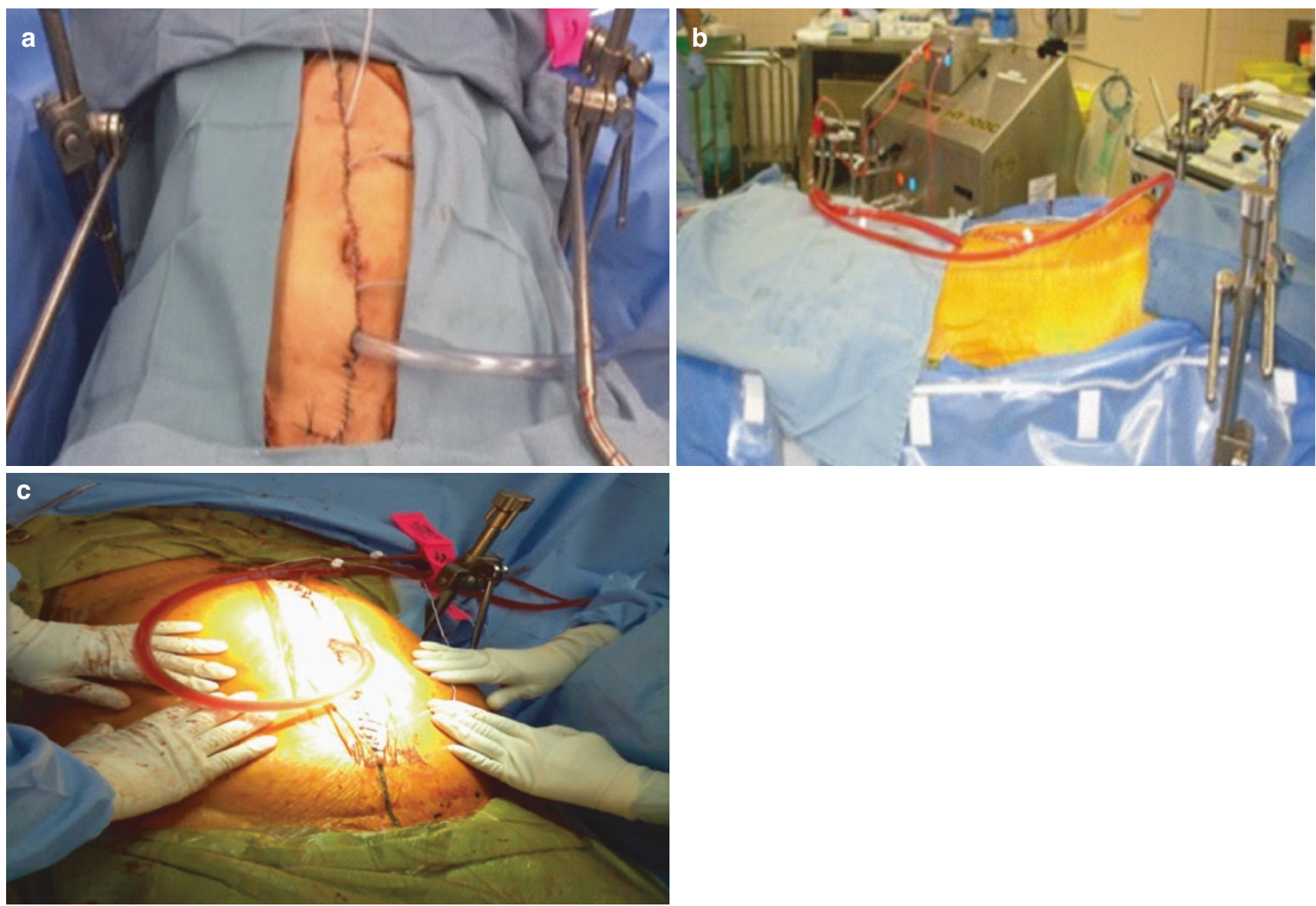

Fig. 23.13 The closed technique. (a, b) The abdomen is temporarily closed with cannulas, temperature probes, and pressure probes incorporated. (c) The abdomen is gently agitated

See Fig. 23.13. The abdomen undergoes gentle external agitation to promote fluid circulation and even distribution of the perfusate. The closed technique requires a larger volume of perfusate and a higher abdominal pressure [2]. This may improve perfusate drug penetration into malignant cells [2]. At the end of HIPEC, the cavity is drained and laparotomy incision reopened; anastomoses are then performed [2].

At our institution we perform a 90-minute perfusion with mitomycin $\mathrm{C}$ at a $42{ }^{\circ} \mathrm{C}$ inflow temperature for colorectal and appendiceal cancer and a 60-minute perfusion when using cisplatin for peritoneal mesothelioma, gastric, and ovarian cancer.

\section{Chemotherapeutic Drugs}

The drug chosen as the chemoperfusant should pose demonstrable activity against the malignancy being treated. The drug must also be directly cytotoxic; drugs needing systemic metabolization into their active form are not appropriate for use with HIPEC [38]. The ideal agent will possess direct cytotoxic activity synergistic with heat, lack local toxicity, without systemic spread, or systemic toxicity [38]. Tumor specificity should be considered: Previous responses to systemic agents may indicate tumor sensitivity or resistance to intraperitoneal agents. Toxicities of the drug chosen is influenced by the drug concentration to the maximal plasma drug concentration [38]. This creates a concentration-time curve gradient and the area under the curve helps dictate maximal doses [38].

The intraperitoneal route will deliver high regional concentrations with minimal systemic effect due to the "peritoneal-plasma" barrier [38]. This barrier maintains minimal displacement of the drug from the peritoneum to the plasma [38]. Limited and delayed absorption through the peritoneum is more pronounced with high-molecular weight 
molecules; therefore these drugs are more favorable for use in HIPEC [38]. Additionally, any drug that is absorbed into the visceral peritoneum will be drained via the portal system and undergo first pass metabolism in the liver, therefore inactivating the drug and minimizing systemic exposure [38]. Renal excretion of the metabolites is usually rapid. The most common presentation of systemic toxicity is bone marrow suppression [38].

Intraperitoneal drug concentration and exposure to the drug are the two biggest determinants that affect treatment [38]. Drug concentration refers to concentration in the peritoneum or tumor cells; concentration of drug in the perfusate fluid is of less importance [38]. Increased local concentration in tissues will improve penetration, and, although this is difficult to measure, the depth of penetration is estimated to be 2-5 mm [38].

Heat alone has a direct antitumor effect. Application of heat causes protein denaturation, impaired DNA repair, inhibition of oxidative metabolism causing cellular acidity, lysosomal activation, and increased apoptosis [2]. Heat shock proteins may limit these direct hyperthermic effects.

The combination of hyperthermia (temperatures above $39-40{ }^{\circ} \mathrm{C}$ ) and neoplastic $\operatorname{drug}(\mathrm{s})$ results in exponential increase in cytotoxic effect [38]. This is dependent upon multiple factors: increased uptake into malignant cells, increased membrane permeability, improved membrane transport, alteration of drug metabolism (decreased adenosine triphosphate transporters allowing drug accumulation), excretion, drug penetration, drug action, and inhibition of repair mechanisms [2, 38]. Heat stability of the drug is a requirement. We will discuss drugs used for each malignancy in the following sections.

In our institution we use mitomycin C (40 mg dose, given in two doses, $30 \mathrm{mg}$ at time zero/10 $\mathrm{mg}$ at time 60 minutes) for appendiceal and colorectal primaries. For gastric cancer, ovarian cancer, and mesothelioma, we use a combination of cisplatin and doxorubicin. In patients with recurrence from appendiceal and colorectal cancer who present and are candidates for a second debulking and HIPEC, we use melphalan (60 mg/m² for 60 minutes) and have had favorable, safe results.

\section{Appendiceal Cancer}

Cancer of the appendix is rare, with approximately $1 \%$ of appendectomy specimens harboring malignancy. Approximately 200-1000 new cases are reported each year, which correlates to 0.12 cases per $1,000,000$ of population [2]. Adenocarcinoma was diagnosed in approximately $65 \%$ of new cases. Traditionally, these patients were treated with systemic chemotherapy and some debulking procedures. This would fail to eradicate the microscopic disease and recurrences would occur in more than 90\% [2].

Prognosis is determined by histologic grade, tumor biology, age, functional status, and extent of disease at diagnosis [2]. Patients may present with copious intraabdominal mucin_-pseudomyxoma peritonei (any primary tumor with copious intraperitoneal mucin production) [2]. However, patients may present without mucin and demonstrate solid peritoneal disease that shows minimal differences from other gastrointestinal malignancies [2]. Sugarbaker and his colleagues first described a new approach in 1980 with CRS combined with hyperthermic intraperitoneal chemotherapy, which has now become the standard of care for treatment of peritoneal dissemination from appendiceal neoplasm [2].

Patients will be diagnosed either inadvertently after surgery or with late systemic or peritoneal disease [2]. These malignancies are classified as either "low grade" or "high grade"; however, there is documentation of differentiation of low-grade malignancies into high-grade lesions in about $16 \%$ of patients [2]. This suggests that these malignancies lie on a spectrum rather than definitive categories. Pattern of spread is related to the grade of disease [2].

Luminal obstruction, usually by mucin, is the first step in disease dissemination of low-grade tumors (Fig. 23.14). Excessive mucin production occludes the lumen, which increases pressure and causes perforation of the appendix with peritoneal dissemination of mucin and tumor cells. Low-grade lesions are associated with implantation and spread along the peritoneal surface in a predictable sequence: right lower quadrant, the pelvis, the right upper quadrant, and finally throughout the abdomen [2]. Distant or lymphatic metastases occurs in less than $10 \%$ of cases [2].

Most centers use mitomycin $\mathrm{C}$ for appendiceal tumors. Mitomycin $\mathrm{C}$ has good activity against gastrointestinal malignancies as an alkylating antibiotic [38]. It has acceptable tumor penetration $(2-5 \mathrm{~mm})$ and an intraperitoneal to plasma drug area under the curve (AUC) ratio of 13-80, indicating good pharmacokinetics and low systemic toxicity [38]. Oxaliplatin has been used in high doses over short intervals (30 minutes). In some institutions, systemic 5-fluorouracil and leucovorin are simultaneously administered to enhance oxaliplatin therapy [38]. There is rapid absorption of the drug into the tumor, although with a low AUC: 13 [38].

Outcomes vary depending on histology, the extent of peritoneal seeding, and comorbidites [2]. Mucin- 

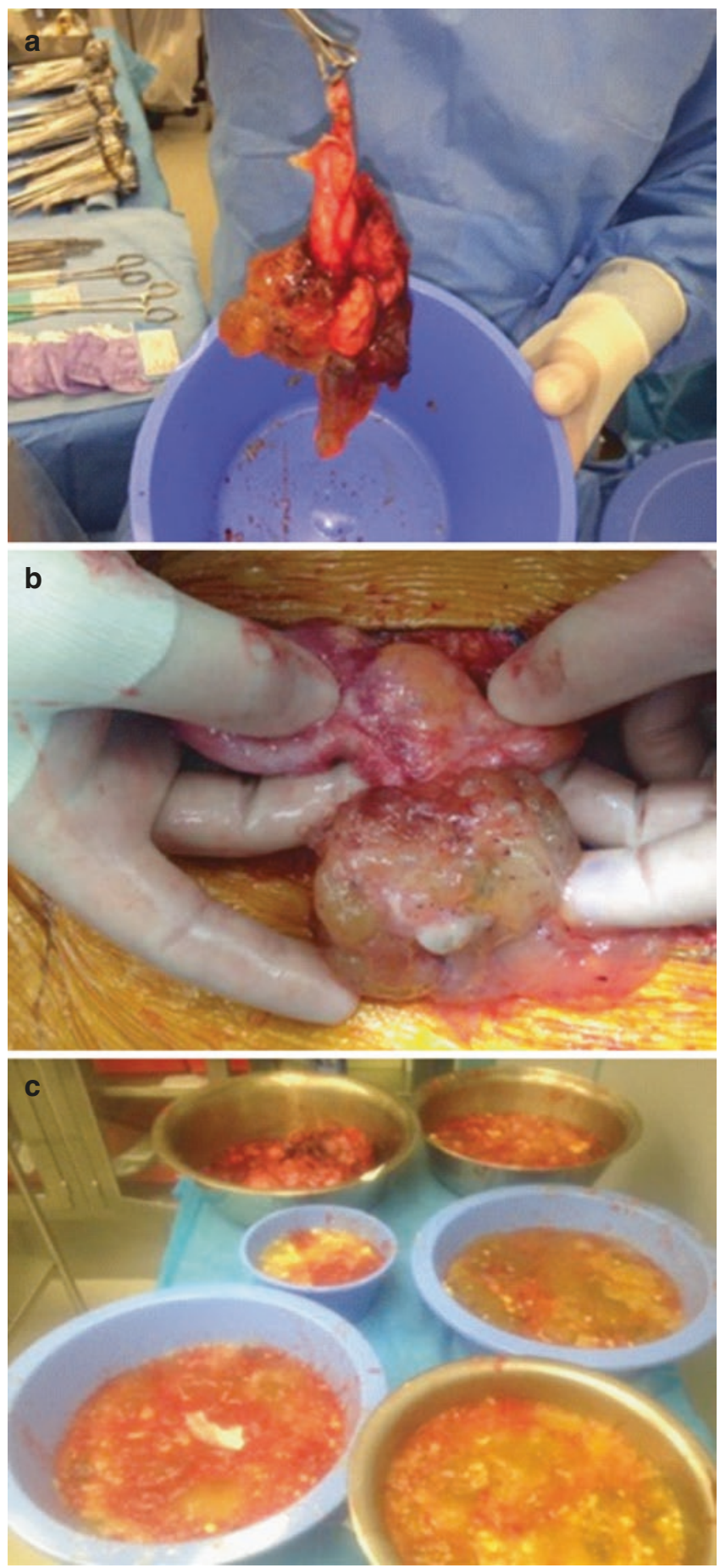

Fig. 23.14 (a, b) Demonstrating the appendix with tumor and mucin production, and (c) mucinous fluid from a patient with a low-grade mucinous neoplasm
Table 23.7 General principles for treatment of low- and high-grade appendiceal mucinous neoplasms

\begin{tabular}{l|l|l}
\hline Histology & LAMN & High-grade \\
\hline Debulking and HIPEC & Yes & Yes \\
\hline Systemic chemotherapy & No (usually) & Yes \\
\hline Right colectomy & No & Yes \\
\hline Median survival & $\sim 10$ years & $\sim 2$ years \\
\hline LN/distant metastasis & No & Yes \\
\hline
\end{tabular}

$L A M N$ low-grade appendiceal mucinous neoplasm, HIPEC hyperthermic intraperitoneal chemotherapy, $L N$ lymph node

producing tumors generally have a more predictable clinical course (peritoneal dissemination) and a better response to therapy [2].

In our series, and in most throughout the nation, the most common reason for cytoreduction and HIPEC is an appendiceal neoplasm. The grade of the appendiceal tumor is of utmost importance, and in general all lowgrade mucinous neoplasms of the appendix may be treated with debulking and HIPEC if needed. We know that this group of patients, if optimally cytoreduced and administered HIPEC, has the best prognoses with median survival exceeding 10 years in most series. This is highly dependent on the grade of the neoplasm and perhaps even the molecular profile of the tumor, as even low-grade tumors with certain molecular mutations may behave aggressively. High-grade neoplasms, as defined by Misradji, can behave as an aggressive invasive malignancy. Thus, the management of highand low-grade appendiceal mucinous tumors may differ depending on the clinical presentation. When we evaluate a patient with a low-grade appendiceal mucinous neoplasm (LAMN), the expected thorough evaluation includes a detailed history and physical examination, laboratory evaluation (including CEA, CA 19-9, and CA-125), review of operative and pathology notes, imaging (CT, MRI, PET), and functional performance status. Some general, although not completely inclusive, principles can help define the course of treatment (Table 23.7).

Furthermore, Sugarbaker and colleagues have recently defined the role of right colectomy and based this on histology of the primary as shown in Fig. 23.15 [39]. 
Fig. 23.15 When should a right colectomy be performed? Proposed algorithm for management of perforated appendiceal epithelial neoplasm. (Reprinted with permission from Sugarbaker [39])

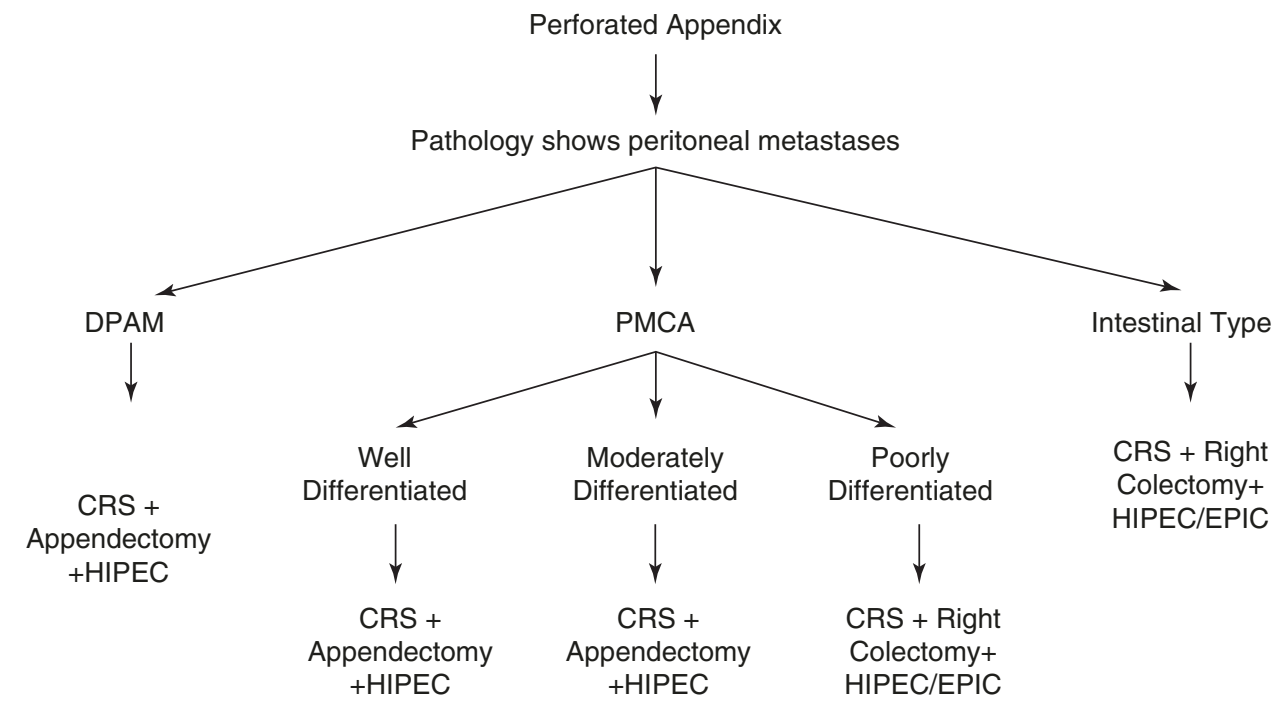

\section{Gastric Cancer}

Gastric cancer accounts for the third most common cause of malignancy-related death ( $8.8 \%$ each year). The presence of peritoneal dissemination with gastric cancer is a sign of advanced tumor stage, progression, and disease recurrence [2]. Risk factors for gastric peritoneal carcinomatosis (GPC) include advanced T stage (serosal involvement), advanced nodal status, tumor size, young age, female gender, signet ring cell histology, and diffuse mixed histology [40]. It is estimated that in $5-43 \%$ of patients who undergo resection with curative intent of the primary tumor, peritoneal dissemination is already present $[2,40]$. Additionally, peritoneal carcinomatosis is the most common synchronous lesion (35\%) [40]. After gastrectomy with D2 lymphadenectomy, peritoneal recurrence occurred in $10-50 \%$ of patients (with peritoneum being the sole site of recurrence in 12-40\%) and distant metastases in $25 \%$ of patients $[40,41]$. This is clearly an indicator of poor prognosis with average time to death of 3-7 months [40, 42]. Systemic chemotherapy regimens only marginally improved survival: 9.5-12 months [40].

Gastric peritoneal carcinomastosis (GPC) occurs with a high frequency due to the tendency of gastric cancer to produce intraperitoneal free cancer cells [40]. These free cells can be found in $24 \%$ of stage I disease and $40 \%$ of stage II or III gastric cancer [40]. The occurrence increases if the malignancy involves the serosa [40]. Traumatic release from surgical manipulation also contributes to intraperitoneal free cancer cells [40]. These cells are released from surrounding lymphatic channels, blood loss within the surgical field, and resection margins [40]. The number of peritoneal lavage specimens positive for malignant cells doubled after gastrectomy (24\% before, $58 \%$ after) [43].
Cells released adhere to exposed surgical surfaces within minutes due to the local release of cytokines, fibrin, and other adhesion molecules [44]. This creates a localized hypoxic environment rendering the cells relatively immune from systemic chemotherapy, thus HIPEC is targeted to these cells.

HIPEC has been used for prophylaxis against PC or adjuvant treatment in gastric cancer. Prophylactic use allows free cells to be washed out with destruction of adhered cells by the synergist effect of chemotherapy and heat [40]. Most of the published literature has been conducted in Asian countries. Some of the earlier studies demonstrate a 3-year survival rate (74\% versus $53 \%$ ) and decreased occurrence of peritoneal recurrence (36\% versus $50 \%$ ) in patients who received prophylactic HIPEC [45]. More studies have demonstrated a survival advantage for patients undergoing HIPEC as prophylactic treatment for PC (Table 23.8) [36, 40, 45-51].

Therapeutic HIPEC has demonstrated survival benefit over CRS alone. Drugs that are commonly used include mitomycin C, cisplatin, and etoposide (in decreasing order). Studies that employed mitomycin $\mathrm{C}$ during their HIPEC demonstrated 5-year survival rate from $11 \%$ to $27 \%$ [5, 52-55].

At our institution we very selectively evaluate patients with gastric cancer and PC for CRS and HIPEC. We use the PCI, determined usually by laparoscopy, and prefer the PCI be less than 10 to consider CRS and HIPEC for patients with GC. There is a role for systemic therapy prior to CRS and HIPEC for these patients and at least disease stability without progression while on chemotherapy should be a prerequisite for consideration of CRS and HIPEC. We advise that these patients only be considered and evaluated at highvolume centers that have a demonstrated experience with complex CRS and HIPEC. 
Table 23.8 Studies of prophylactic hyperthermic intraperitoneal chemotherapy (HIPEC) in gastric cancer

\begin{tabular}{|c|c|c|c|c|}
\hline Reference & Type of study & Drug used & Survival (HIPEC vs no HIPEC) & $\begin{array}{l}\text { Peritoneal recurrence } \\
\text { (HIPEC vs no HIPEC) }\end{array}$ \\
\hline Koga et al. [45] & $\begin{array}{l}\text { Randomized } \\
\text { controlled trial (RCT) }\end{array}$ & Mitomycin C (MMC) & 30 mo: $83 \%$ vs $67 \%$ & N/A \\
\hline Hamazoe et al. [46] & RCT & MMC & $\begin{array}{l}5 \text { year: } 64 \% \text { vs } 52 \% \\
\text { Median survival: } 77 \text { mo vs } 66 \\
\text { mo }\end{array}$ & $39 \%$ vs $59 \%$ \\
\hline Fujimura et al. [36] & RCT & MMC and cisplatin & 3 year: $68 \%$ vs $23 \%$ & $9 \%$ vs $22 \%$ \\
\hline Ikeguchi et al. [47] & RCT & MMC & 5 year: $51 \%$ vs $46 \%$ & $35 \%$ vs $40 \%$ \\
\hline Fujimoto et al. [48] & $\mathrm{RCT}$ & MMC & $\begin{array}{l}2 \text { year: } 88 \% \text { vs } 77 \% \\
4 \text { year: } 76 \% \text { vs } 58 \% \\
8 \text { year: } 62 \% \text { vs } 49 \% \\
\end{array}$ & $1.4 \%$ vs $23 \%$ \\
\hline Hirose et al. [49] & $\begin{array}{l}\text { Prospective case } \\
\text { control }\end{array}$ & $\begin{array}{l}\text { MMC, cisplatin, and } \\
\text { etoposide }\end{array}$ & $\begin{array}{l}3 \text { year: } 49 \% \text { vs } 29 \% \\
5 \text { year: } 39 \% \text { vs } 17 \% \\
\text { Median survival: } 33 \text { mo vs } 22 \\
\text { mo }\end{array}$ & 26 vs $45 \%$ \\
\hline Yonemura et al. [50] & RCT & MMC and cisplatin & 5 year: $61 \%$ vs $42 \%$ & 13 vs $15 \%$ \\
\hline Kim et al. [51] & $\begin{array}{l}\begin{array}{l}\text { Prospective controlled } \\
\text { study }\end{array} \\
\end{array}$ & MMC & 5 year: $33 \%$ vs $27 \%$ & $7.6 \%$ vs $25 \%$ \\
\hline
\end{tabular}

Modified from Seshadri and Glehen [40]

\section{Colorectal Cancer}

Patients who present with metastatic disease have 5-7\% incidence of PC with one-third of those presenting with isolated PC [2]. The presence of PC worsens prognosis. Historically, the prognosis was no greater than 6 months if no intervention was undertaken [2]. The most common systemic chemotherapeutic regimens employed include leucovorin/5-fluorouracil/oxaliplatin (FOLFOX) or leucovorin/5-fluorouracil/irinotecan (FOLFIRI). The North American N9741 and N9841 trials demonstrated a median survival of 12.7 months for patients with PC and 17.6 months for patients without PC [2]. The 5-year survival rate was $4.1 \%$ and $6 \%$ for the groups [2]. The addition of newer agents bevacizumab and cetuximab has demonstrated additional survival. Median survival has been prolonged 3-6 months [2]. Saltz et al. reported a median survival of patients receiving FOLFOX + bevacizumab was 21.3 months $[2,56]$.

Peritoneal dissemination treated with surgery alone has demonstrated no survival benefit if complete cytoreduction cannot be carried out [2]. Studies demonstrated that median survival of patients who underwent incomplete resection ranges from 6.3 to 15 months, while patients who had systemic chemotherapy alone had a mean survival of 8-17 months [2].

When the disease is limited, complete cytoreduction is feasible. Prior to the use of HIPEC, patients who had a good performance status with limited disease demonstrated a median survival of 25 months and a 5-year survival of $22 \%$ when complete cytoreduction could be performed [57]. In the same study, median survival for patients after systemic chemotherapy alone was 18 months [57]. It is generally agreed upon that a PCI $<20$ is possibly amenable to surgical resection. When a patient presents with a PCI $>20$, palliative surgery may be considered only to relieve symptoms [11, 12].

When performed at an experienced center, the 5-year survival rate for those patients who received CRS + HIPEC was $42-51 \%$ with a median survival of $33-41$ months. This is compared to $13 \%$ for those who received only chemotherapy [2]. Additional studies show that after 5 years (from the date of their last treatment) $16 \%$ of patients had no recurrence and were considered "cured" [2]. However, the new novel targeted agents have allowed patients with peritoneal carcinomatosis from colorectal cancer to achieve a median survival in some cases up to 30 months with combination systemic therapy alone.

Both mitomycin $\mathrm{C}$ and oxaliplatin have been investigated for use during HIPEC for PC due to colorectal carcinomatosis [38]. Oxaliplatin used in short durations (30 minutes) at high concentrations appears to be well tolerated; systemic intravenous 5-fluorouracil and leucovorin are concurrently administered to enhance oxaliplatin activity [38]. Although with good initial results, recent data suggests that mitomycin $\mathrm{C}$ may be a better agent for HIPEC due to colorectal carcinoma with PC [58]. This was demonstrated in patients with low burden of disease and favorable pathology. Interestingly, in patients with unfavorable histology and a high burden of disease, a nonsignificant better overall survival was demonstrated when oxaliplatin was used [29]. More prospective studies are needed. There are a few studies demonstrating use of irinotecan for HIPEC [38]. This drug, which is activated through liver metabolization, has demonstrated high intraperitoneal concentrations suggesting possible activity against PC [38].

However, studies that employed irinotecan with oxalplatin demonstrated increased morbidity without survival 
advantages [38]. Additional studies are needed before regular use of irinotecan. Melphalan has significant effect against a wide range of gastrointestinal malignancies [38]. Its synergist effect with heat and favorable tissue distribution makes it a good option for recurrent malignancies or salvage procedures [38].

Perhaps the most exciting change that has occurred in recent months is the addition of CRS and HIPEC into the National Comprehensive Cancer Network (NCCN) guidelines. Specifically, the Version 2.2017 guidelines suggest that for patients with synchronous abdominal/peritoneal metastases, "complete cytoreductive surgery and/or intraperitoneal chemotherapy can be considered in experienced centers for select patients with limited PC for whom R0 resection can be achieved" [59]. This is the first mention of CRS and HIPEC in the NCCN guidelines and evidence that this may be a viable option accepted by the medical community for patients with PC from CRC.

\section{Mesothelioma}

Malignant peritoneal mesothelioma (MPM) accounts for $30 \%$ of all malignant mesothelioma cases $[2,60,61]$. It is an aggressive tumor that has dismal survival of 6-12 months without intervention [61]. Other sites for malignant mesothelioma include the pleura (most common), the pericardium, and the tunica vaginalis, with each site demonstrating individual epidemiology [2]. MPM is most common in females with a mean age of 65-66 years [2, 61].

It is suggested that the development of peritoneal mesothelioma possibly occurs through exposure to asbestos. Asbestos fibers trigger a foreign body reaction with subsequent inflammatory response. The ferritin heavy chain present in the asbestos fibers creates reactive oxygen species and reactive nitrogen species. The accumulation of these actions results in genetic disruption leading to mutations in the tumor suppressor gene BAP-1 [60]. The asbestos is inhaled, expectorated, and swallowed. In cases without asbestos exposure, an oncogenic virus (i.e., the simian vacuolating virus-SV40) has been implicated, although more data is needed to affirm any relationship [2].

MPM is a locoregional disease, meaning it has a tendency to remain in the abdomen throughout disease progression $[60,61]$. It has a highly variable rate of progression [60]. When disease is found outside of the abdomen it is most often by direct extension, trans-diaphragmatic lymphatic, or extra-abdominal lymph node metastasis [61].

Three histologic subtypes of MPM exist: epitheliod (multicystic subtype), sarcomatoid, and mixed/biphasic type. Epitheliod is the most common. Only with the use of immunohistochemical antibodies can the three types be differentiated [60]. Calretinin, cytokeratin 5/6, and vimentin are most
Table 23.9 A proposed staging system for malignant peritoneal mesothelioma (MPM)

\begin{tabular}{l|l|l|l|l}
\hline & $\begin{array}{l}\text { Peritoneal } \\
\text { carcinomatosis index } \\
\text { (stratified into } \\
\text { quartiles as a } \\
\text { surrogate for tumor } \\
\text { stages) }\end{array}$ & $\begin{array}{l}\text { Tumor } \\
\text { stage }\end{array}$ & $\begin{array}{l}\text { Node stage } \\
\text { (extra- } \\
\text { abdominal } \\
\text { nodal } \\
\text { metastases) }\end{array}$ & $\begin{array}{l}\text { Metastasis } \\
\text { stage } \\
\text { (extra- } \\
\text { abdominal } \\
\text { metastases) }\end{array}$ \\
\hline I & $1-10$ & 1 & 0 & 0 \\
\hline II & $11-20$ & 2 & 0 & 0 \\
\hline & $21-30$ & 3 & 0 & 0 \\
\hline III & $21-39$ & 4 & $0-1$ & $0-1$ \\
\hline & $1-39$ & $1-4$ & 1 & 1 \\
\hline
\end{tabular}

Modified from Alexander Jr and Burke [60]

commonly used [60]. At least two stains must be used to confirm MPM. Some studies suggest an elevated CA-125 tumor marker; however, this is unreliable and best used to monitor for recurrent disease [2, 60].

Staging of MPM cannot be carried out by conventional tumor-node-metastasis (TNM) staging due to its propensity to remain intra-abdominal. A proposed staging system is outlined in Table 23.9 [2, 60].

The best observed outcomes are for those with CCR 0-1. Median overall survival ranges from 30 to 92 months and was associated with epithelioid type (multicystic subvariant) absence of lymph node metastasis, achievement of CCR 0/1, and use of HIPEC [2, 60, 61]. The 1-, 3-, and 5-year survival rates after CRS with HIPEC are 70\%, 60\%, and 41-64\% [2, $60,61]$. Age also affects survival, with a 5-year survival of $89 \%$ for those younger than 55 years versus $15 \%$ for those 55 years of age or older [61]. Features most predictive of poor prognosis include sarcomatoid growth pattern, degree of tissue invasion into stroma, fat or adjacent structures, and CCR of 2 or greater [61].

There are multiple chemotherapeutic agents reported to be effective against MPM. These include cisplatin, doxorubicin, mitomycin $\mathrm{C}$, and docetaxel $[2,38]$. These drugs have been used as solo regimens or in combination. The most common being cisplatin, doxorubicin, and mitomycin C. Doxorubicin has multiple features making it a good choice for HIPEC: high molecular weight, no dose-limiting toxicity (when used intraperitoneal), tumor sequestration, and thermal enhancement [38]. A point to highlight is the tumor sequestration feature of this drug. Doxorubicin will preferentially infiltrate tumor cells, despite underlying pathology [38]. It makes predicting intra-tumor concentration based on sample of peritoneal fluid difficult; however, this may result in improved efficacy of intraperitoneal administration [38]. More research is needed to discover the mechanism. The other commonly used drugs, cisplatin and mitomycin $\mathrm{C}$, have been discussed elsewhere. Pemetrexed is another drug being studied for user in MPM. Pemetrexed has excellent systemic activity against mesothelioma and may be a poten- 
tial agent [38]. Currently, there is no evidence suggesting a survival advantage with use of any specific drug.

\section{Ovarian Cancer}

Ovarian cancer of epithelial origin (EOC) has a worldwide incidence of more than 200,000 per year and is responsible for 125,000 deaths annually [2]. Five-year survival is less than $50 \%$ for most who present with disease that has already spread outside of the pelvis $(50.2 \%$ classified as Stage III disease by The International Federation of Gynecology and Obstetrics) [2]. Approximately 13\% present with distant metastasis (Stage IV).

EOC remains confined to the peritoneal cavity and retroperitoneal lymph nodes for most of its disease course [2]. For many years it was thought to arise from epithelial covering of the ovarian. However, it is now thought to more likely arise from the distal fallopian tube epithelium that adheres to the ovary during ovulation [62]. Survival for EOC is poor with a 5-year survival rate of approximately $49 \%$ [2].

A distinct subtype of EOC is low malignant potential (LMP) tumors. Often referred to as borderline or atypical tumors, LMP tumors occur at an earlier stage, younger age with a better prognosis, and less aggressive histology [2]. Peritoneal carcinomatosis is a feature of advanced disease and is associated with a poorer prognosis [2].

Broadly, the natural history of EOC can be divided according to treatment time points: front-line, front-line failure, consolidation, and recurrent disease [2]. Front-line failure is considered persistent disease at the end of front-line treatment [2]. In contrast, consolidation treatment is given following a complete response to front-line therapy [2]. Prognosis is determined by response to a platinum-based chemotherapy: platinum sensitive or platinum resistant [2]. Those with disease that recurs greater than 6 months after platinum therapy are considered sensitive, while those who recur less than 6 months are considered resistant [2].

Front-line treatment consists of CRS with platinum- and taxane-based systemic chemotherapy [2]. Prognosis is determined by the amount of residual disease after CRS, with most gynecologic oncology surgeons aiming to remove all visible disease $(<1 \mathrm{~cm})$ [2]. Some argue that there may be a greater chance for complete CRS if chemotherapy is administered and used to decrease the volume of disease and ascites [2]. This may improve preoperative performance status (PS), shorten the length of operation, and decrease operative morbidity [2]. Some studies demonstrated a survival advantage for patients who underwent initial CRS followed by chemotherapy (versus initial neoadjuvant chemotherapy), while a European study showed similar survival for women with Stage IIIC and IV disease [2, 63, 64]. Patients who had suboptimal CRS ( $>2 \mathrm{~cm}$ residual disease) had shorter progression-free survival (PFS) and overall survival (OS) after delivery of a platinum- and taxane-based chemotherapy versus those who had optimal CRS ( $<1 \mathrm{~cm}$ residual disease) [2]. Those with suboptimal CRS had PFS of 14.1 months and OS of 26.3 months, while those with optimal CRS had PFS of 18.3-23.8 months and OS of 48.7-65.6 months [2]. Studies have suggestive that survival may be up to 106 months if no visible disease remains at the end of CRS [2]. The addition of bevacizumab to standard chemotherapeutic regimens, for front-line treatment, has shown no significant increases in PFS or OS [2].

Prior to 2010 there were no consensus guidelines on the use of HIPEC as treatment for front-line, front-line failure, consolidation, or recurrent disease [2]. The creation of the Hyperthermic Intraperitoneal Chemotherapy in Ovarian Cancer Registry (HYPERO) has allowed the pooling of data and publication of multi-institutional studies on the use of CRS + HIPEC in ovarian cancer [65-67]. The initial report from HYPERO in 2010 demonstrated no OS and 2 year PFS with use of HIPCE versus conventional treatment [2, 66, 67]. More recent data published demonstrates median OS of 25.7-30.3 months with 2-, 5-, and 10-year OS of 49.1\%, $23-25.4 \%$, and $14.3 \%$ in both treatment naïve and recurrent EOC $[68,69]$. Factors significant for increased survival were sensitivity to platinum response, completeness of CCR, carboplatin alone or a combination of two or more chemotherapy agents used and duration of hospital stays of 10 days or less, Eastern Cooperative Oncology Group performance status, and preoperative serum and albumin $[68,69]$.

\section{Postoperative Course, Complications, and Long-Term Surveillance}

Previously diagnosed comorbidities are present in $18 \%$ of patients undergoing major oncologic resection. These morbidities increase the risk of acute medical complications (odds ratio 3.7), in-hospital mortality (OR 3.6), hospital costs, postoperative complications (OR 3.9), and increased complication severity (OR 3.6) [8]. The risk of 30-day postoperative mortality increased with increasing age, American Society of Anesthesiologists (ASA) score $>3$, presence of pulmonary disease, serum albumin $<2.5 \mathrm{mg} / \mathrm{dL}$ and receiving $>1$ unit red blood cell transfusion intraoperative, liver disease, renal disease, sepsis, steroid use, weight loss, bleeding disorder, obesity, cardiac morbidity, and do not resuscitate status $[70,71]$. Risk factors of increased length of stay include age $>75$ years, male gender, current smoker, dependent functional capacity, preoperative serum sodium $<135 \mathrm{mmol} / \mathrm{L}$, serum albumin $<2.5 \mathrm{mg} / \mathrm{dL}$, white blood cell count $>11,000$ cells $/ \mathrm{mm}^{3}$, and hematocrit $<37 \%$ [71].

Once thought to be an extremely morbid procedure with high mortality, the consensus guidelines published by the American Society of Peritoneal Surface Malignancies has helped improve 
perioperative morbidity and mortality [72]. Perioperative morbidity has been classified according to the Clavien-Dindo scale in some accounts, and this data demonstrates grade III complication rates occurring in $26-33 \%$ of cases and grade IV complications occurring in 12-26\% of cases [18-20]. Average length of operation ranged from 433 to 470 minutes [18-20]. Recent data demonstrated a low morbidity and zero mortality rate for complex oncologic resections: grade I, 7\%; grade II, 33\%; grade III, 9\%; and grade IV, $2 \%$ [73]. There was $0 \%$ mortality at $0,30,60$, and 90 days postoperatively $(n=54)$ [73]. Length of stay was 8.2 days with 30-day readmission rate of $6 \%$ [73].

Postoperative surveillance is difficult and thus there is no consensus on optimal surveillance methods. The use of serial tumor markers is difficult; one study suggested that if preoperative CEA and CA19-9 are elevated, then an elevated postoperative CA19-9 was predictive of recurrence [74]. This does not predict the volume of disease, disease stabilization, full or partial responses [75]. Magnetic resonance imagining can detect tumor recurrence earlier than tumor markers for appendiceal neoplasms [76]. Postoperatively, patients underwent surveillance MRI and tumor markers every 6 months; recurrence was identified on average 13 months postoperatively [76]. Of the patients identified by MRI, 37\% were identified to have normal tumor marker levels [76]. Tumor markers identified half as many patients with disease recurrence compared to MRI [76]. More studies are needed to evaluate imaging modalities for other pathologies. Generally, imaging should be performed at 3, 6, and 12 months postoperatively and yearly after that.

There is some data supporting the practice of a second look operation with patients who have undergone complete CRS + HIPEC with CRC and high-risk features [2]. Elias and colleagues carried out a prospective study to analyze outcomes of a second-look laparotomy 1 year after initial CRS + HIPEC [4]. All patients included in this group were found to have high-risk features at the original operation: previous-limited PC, resected ovarian metastases, and a perforated primary lesion [4]. Patients were asymptomatic and without evidence of disease (tumor markers, clinical exam, MRI, CT, PET scan) [4]. When the second look laparotomy was performed, $55 \%$ of patients were found to have visible PC [4]. These patients underwent CRS + HIPEC, and 12 months from the second look operation $50 \%$ were found to be disease free [4]. Although more trials and data are needed, this demonstrates that a planned second look laparotomy with intent for complete CRS + HIPEC may be of some benefit to select patients.

Long-term quality of life (QoL) and recovery remains largely unknown in this patient population. Traditionally associated with significant morbidity, studies now demonstrate lower morbidity rates, which may translate into improved quality of life for patients. There are few studies that explore the QoL in the postoperative period. The major- ity of these studies are single-center reviews that use validated questionnaires not specific for CRS + HIPEC [77]. When administered at the time of surgery and $3,6,9$, or 12 months postoperatively, most patients demonstrate a return to an acceptable performance status between 3 and 24 month, with a return to baseline at 6-24 months postoperatively [77]. Studies demonstrate a lag in recovery of mental health while social functioning returned to baseline status 3 months postoperatively [77]. With such quick returns to an acceptable PS, one may extrapolate that patients may continue to improve beyond baseline at 6,9 , or 12 months postoperatively [77].

\section{Conclusion}

In this chapter we have attempted to give a summary of the diagnosis, management, and treatment of peritoneal malignancies. This is a rapidly evolving area of interest for surgical and medical oncologists throughout the world. Rigorous patient evaluation and selection we feel is a key to successful management of patients with PC from any malignancy. The disease burden and the histology of the primary tumor and metastases we propose are of extreme importance in determining whether patients are candidates for this approach. Finally, the performance status and lack of extra-abdominal disease are of utmost importance in evaluating this group of patients for CRS and HIPEC.

In patients with PC from appendiceal, colorectal, mesothelioma, ovarian, and primary peritoneal cancer there is a substantial amount of data and support for at least an evaluation of these patients in a center with an experienced peritoneal surface malignancy team. For other primary gastrointestinal cancers - such as gastric, pancreatic, hepatobiliary, and other more uncommon disease-there is less data to support the routine use of CRS and HIPEC. In particular, these patients should be evaluated in centers with a multidisciplinary team that has significant experience.

There continues to be new developments in this field, and it would seem the role of heated and even normothermic intraperitoneal chemotherapy for patients with PC will continue to evolve in an effort to improve the quality of life and survival of these patients faced with an extremely challenging disease.

\section{References}

1. Zhu Y, Hanna N, Boutros C, Alexander HR Jr. Assessment of clinical benefit and quality of life in patients undergoing cytoreduction and Hyperthermic Intraperitoneal Chemotherapy (HIPEC) for management of peritoneal metastases. J Gastrointest Oncol. 2013;4(1):62-71.

2. Esquivel J. Surgical oncology clinics of North America: treatment of peritoneal malignancies. Surg Oncol Clin North Am. 2012;21:4. 
3. Kuijpers AM, Mirck B, Aalbers AG, Nienhuijs SW, de Hingh IH, Wiezer MJ, et al. Cytoreduction and HIPEC in the Netherlands: nationwide long-term outcome following the Dutch protocol. Ann Surg Oncol. 2013;20(13):4224-30.

4. Elias D, Goéré D, Di Pietrantonio D, Boige V, Malka D, KohnehShahri N, et al. Results of a systematic second-look surgery in patients at high risk of developing colorectal peritoneal carcinomatosis. Ann Surg. 2008;247(3):445-50.

5. Glehen O, Schreiber V, Cotte E, Sayag-Beaujard AC, Osinsky D, Freyer G, et al. Cytoreductive surgery and intraperitoneal chemohyperthermia for peritoneal carcinomatosis arising from gastric cancer. Arch Surg. 2004;139:20-6.

6. Sugarbaker PH. Management of peritoneal surface malignancy using intraperitoneal chemotherapy and cytoreductive surgery: manual for physicians and nurses. Grand Rapids: The Ludann Company; 1998.

7. Oken MM, Creech RH, Tormey DC, Horton J, Davis TE, McFadden ET, et al. Toxicity and response criteria of the Eastern Cooperative Oncology Group. Am J Clin Oncol. 1982;5:649-55.

8. Sinha P, Kallogjeri D, Piccirillo JF. Assessment of comorbidities in surgical oncology outcomes. J Surg Oncol. 2014;110(5):629-35.

9. Ford J, Coughlin KR, Van Dorp D, Berri RN. Validation of the American College of Surgeons (ACS) National Surgical Quality Improvement Program (NSQIP) risk calculator to estimate serious complications in major gastrointestinal oncologic resection. J Am Coll Surg. 2015;221(4 Suppl. 2):e135-6.

10. Valle M, Federici O, Garofalo A. Patient selection for cytoreductive surgery and hyperthermic intraperitoneal chemotherapy, and role of laparoscopy in diagnosis, staging and treatment. Surg Oncol Clin N Am. 2012;21(4):515-31.

11. Harmon RL, Sugarbaker PH. Prognostic indicators in peritoneal carcinomatosis from gastrointestinal cancer. Int Semin Surg Oncol. 2005;2(1):3.

12. Pelz JO, Stojadinovic A, Nissan A, Hohenberger W, Esquivel J. Evaluation of a peritoneal surface disease severity score in patients with colon cancer with peritoneal carcinomatosis. J Surg Oncol. 2009;99(1):9-15.

13. Keating NL, Landrum MB, Lamont EB, Bozeman SR, Shulman $\mathrm{LN}, \mathrm{McNeil} \mathrm{BJ}$. Tumor boards and the quality of cancer care. J Natl Cancer Inst. 2013;105(2):113-21.

14. Elias D, Gilly F, Boutitie F, Quenet F, Bereder JM, Mansvelt B, et al. Peritoneal colorectal carcinomatosis treated with surgery and perioperative intraperitoneal chemotherapy: retrospective analysis of 523 patients from a multicentric French study. J Clin Oncol. 2010;28(1):63-8.

15. Sugarbaker PH. Management of peritoneal surface malignancy: the surgeons role. Langenbacks Arch Surg. 1999;384:576-87.

16. Yoon W, Alame A, Berri R. Peritoneal surface disease severity score as predictor of resectability in treatment of peritoneal surface malignancies. Am J Surg. 2014;207(3):403-7; discussion 406-7.

17. Esquivel J, et al. ASPSM multi-institution evaluation of the peritoneal surface disease severity score in 1,013 patients with colorectal cancer with peritoneal carcinomatosis. Ann Surg Oncol. 2014;21:4195-201.

18. Tsilimparis N, Bockelmann C, Raue W, Menenakos C, Perez S, Rau B, et al. Quality of life in patients after cytoreductive surgery and hyperthermic intraperitoneal chemotherapy: is it worth the risk? Ann Surg Oncol. 2013;20(1):226-32.

19. Tan WJ, Wong JF, Chia CS, Tan GH, Soo KC, Teo MC. Quality of life after cytoreductive surgery and hyperthermic intraperitoneal chemotherapy: an Asian perspective. Ann Surg Oncol. 2013;20(13):4219-23.

20. Glockzin G, Schlitt HJ, Piso P. Peritoneal carcinomatosis: patients selection, perioperative complications and quality of life related to cytoreductive surgery and hyperthermic intraperitoneal chemotherapy. World J Surg Oncol. 2009;7:5.
21. Esquivel J, Angulo F, Bland RK, Stephens AD, Sugarbaker $\mathrm{PH}$. Hemodynamic and cardiac function parameters during heated intraoperative intraperitoneal chemotherapy using the open "coliseum technique”. Ann Surg Oncol. 2000;7(4):296-300.

22. Turaga K, Levine E, Barone R, Sticca R, Petrelli N, Lambert L, et al. Consensus guidelines from the American Society of Peritoneal Surface Malignancies on standardizing the delivery of hyperthermic intraperitoneal chemotherapy (HIPEC) in colorectal cancer patients in the United States. Ann Surg Oncol. 2014;21:1501-5.

23. Joshi GP. Intraoperative fluid restriction improves outcomes after major electives gastrointestinal surgery. Anesth Analg. 2005;101(2):601-5.

24. Bailey JM. Dopamine: one size does not fit all. Anesthesiology. 2000;92(2):303-5.

25. Thix CA, Königsrainer I, Kind R, Wied P, Schroeder TH. Ventricular tachycardia during hyperthermic intraperitoneal chemotherapy. Anesethsia. 2009;64(10):1134-6.

26. Rueth NM, Murray SE, Huddleston SJ, Abbott AM, Greeno EW, Kirstein MN, et al. Severe electrolyte disturbances after hyperthermic intraperitoneal chemotherapy: oxaliplatin versus mitomycin C. Ann Surg Onc. 2011;18(1):174-80.

27. Esquivel J, Averbach A, Chua T. Laparoscopic cytoreductive surgery and hyperthermic intraperitoneal chemotherapy in patients with limited peritoneal surface malignancies: feasibility, morbidity and outcome in an early experience. Ann Surg. 2011;253(4):764-8.

28. Passot G, Bakrin N, Isaac S. Postoperative outcomes of laparoscopic vs open cytoreductive surgery plus hyperthermic intraperitoneal chemotherapy for treatment of peritoneal surface malignancies. Eur J Surg Oncol. 2014;40(8):957-62.

29. Sugarbaker PH. Cytoreductive surgery using peritonectomy and visceral resections for peritoneal surface malignancy. Transl Gastrointest Cancer. 2013;2(2)

30. Bijelic L, Sugarbaker PH. Cytoreduction of the small bowel surfaces. J Surg Oncol. 2008;97(2):176-9.

31. Halkia E, Efstathiou E, Rogdakis A, Christakis C, Spiliotis J. Digestive fistulas after cytoreductive surgery \& HIPEC in peritoneal carcinomatosis. J BUON. 2015;20(Suppl 1):S60-3.

32. Esquis P, Consolo D, Magnin G, Pointaire P, Moretto P, Ynsa MD, et al. High intraabdominal pressure enhances the penetration and antitumor effect of intraperitoneal cisplatin on experimental peritoneal carcinomatosis. Ann Surg. 2006;244(1):106-12.

33. Esquivel J. Membership. http://www.americansocietypsm.org. Last accessed May 15, 2018.

34. González-Moreno S, González-Bayón LA, Ortega-Pérez G. Hyperthermic intraperitoneal chemotherapy: rationale and technique. World J Gastrointest Oncol. 2010;2(2):68-75.

35. Esquivel J, Sugarbaker PH, Helm CW. Techniques of delivering hyperthermic intraperitoneal chemotherapy. In: Helm CW, Edwards RP, editors. Intraperitoneal Cancer therapy. Current clinical oncology: Springer; 2007.

36. Fujimura T, Yonemura Y, Muraoka K, Takamura H, Hirono Y, Sahara $\mathrm{H}$, et al. Continuous hyperthermic peritoneal perfusion for the prevention of peritoneal recurrence of gastric cancer: randomized controlled study. World J Surg. 1994;18(1):150-5.

37. Glehen O, Cotte E, Kusamura S, Deraco M, Baratti D, Passot G, et al. Hyperthermic intraperitoneal chemotherapy: nomenclature and modalities of perfusion. J Surg Oncol. 2008;98:242-6.

38. De Bree E. Optimal drugs for HIPEC in different tumors. J BUON. 2015;20(Suppl. 1):S40-6.

39. Sugarbaker PH. When and when not to perform a right colon resection with mucinous appendiceal neoplasms. Ann Surg Oncol. 2017;24(3):729-32.

40. Seshadri R, Glehen O. Cytoreductive surgery and hyperthermic intraperitoneal chemotherapy in gastric cancer. World $\mathrm{J}$ Gastroenterol. 2016;22(3):1114-30. 
41. Sasako M, Sano T, Yamamoto S, Kurokawa Y, Nashimoto A, Kurita A, Japan Clinical Oncology Group, et al. D2 lymphadenectomy alone or with paraaortic nodal dissection for gastric cancer. N Engl J Med. 2008;359(5):453-62.

42. D'Angelica M, Gonen M, Brennan MF, Turnbull AD, Bains M, Karpeh MS. Patterns of initial recurrence in completely resected gastric adenocarcinoma. Ann Surg. 2004;240(5):808-16.

43. Yu XF, Ren ZG, Xue YW, Song HT, Wei YZ, Li CM. D2 lymphadenectomy can disseminate tumor cells into peritoneal cavity in patients with advanced gastric cancer. Neoplasma. 2013;60:174-81.

44. Sugarbaker PH, Yu W, Yonemura Y. Gastrectomy, peritonectomy and perioperative intraperitoneal chemotherapy: the evolution of treatment strategies for advanced gastric cancer. Semin Surg Oncol. 2003;21:233-48.

45. Koga S, Hamazoe R, Maeta M, Shimizu N, Murakami A, Wakatsuki T. Prophylactic therapy for peritoneal recurrence of gastric cancer by continuous hyperthermic peritoneal perfusion with mitomycin C. Cancer. 1988;61:223-37.

46. Hamazoe R, Maeta M, Kaibara N. Intraperitoneal thermochemotherapy for prevention of peritoneal recurrences of gastric cancer. Final results of a randomized controlled study. Cancer. 1994;73:2048-52.

47. Ikeguchi M, Kondou A, Oka A, Tsujitani S, Maeta M, Kaibara N. Effects of continuous hyperthermic peritoneal perfusion on prognosis of gastric cancer with serosal invasion. Eur J Surg. 1995;161:581-6.

48. Fujimoto S, Takahashi M, Mutou T, Kobayashi K, Toyosawa T. Successful intraperitoneal hyperthermic chemoperfusion for the prevention of postoperative peritoneal recurrences in patients with advanced gastric cancer. Cancer. 1999;85:529-34.

49. Hirose K, Katayama K, Iida A, Yamaguchi A, Nakagawara G, Umeda S, et al. Efficacy of continuous hyperthermic peritoneal perfusion for the prophylaxis and treatment of peritoneal metastasis of advanced gastric cancer; evaluation by multivariate regression analysis. Oncology. 1999;57:106-14.

50. Yonemura Y, de Aretxabala X, Fujimura T, Fushida S, Katayama $\mathrm{K}$, Bandou $\mathrm{E}$, et al. Intraoperative chemohyperthermic peritoneal perfusion as an adjuvant to gastric cancer: final results of a randomized controlled study. Hepato-Gastroenterology. 2001;48:1776-82.

51. Kim JY, Bae HS. A controlled clinical study of serosa-invasive gastric carcinoma patients who underwent surgery plus intraperitoneal hyperthermo-chemo-perfusion (IHCP). Gastric Cancer. 2001;4(1):27-33.

52. Yonemura Y, Fujimura T, Nishimura G, Falla R, Sawa T, Katayama $\mathrm{K}$, et al. Effects of intraoperative chemohyperthermia in patients with gastric cancer with peritoneal dissemination. Surgery. 1996;119:437-44.

53. Fujimoto S, Takahashi M, Mutou T, Kobayashi K, Toyosawa $\mathrm{T}$, Isawa $\mathrm{E}$, et al. Improved mortality rate of gastric carcinoma patients with peritoneal carcinomatosis treated with intraperitoneal hyperthermic chemoperfusion combined with surgery. Cancer. 1997;79(5):884-91.

54. Yonemura Y, Kawamura T, Bandou E, Takahashi S, Sawa T, Matsuki N. Treatment of peritoneal dissemination from gastric cancer by peritonectomy and chemohyperthermic peritoneal perfusion. Br J Surg. 2005;92:370-5.

55. Glehen O, Gilly FN, Arvieux C, Cotte E, Boutitie F, Mansvelt $\mathrm{B}$, et al. Peritoneal carcinomatosis from gastric cancer: a multiinstitutional study of 159 patients treated by cytoreductive surgery combined with perioperative intraperitoneal chemotherapy. Am Surg Oncol. 2010;17:2370-7.

56. Saltz LB, Clarke S, Díaz-Rubio E, Scheithauer W, Figer A, Wong $\mathrm{R}$, et al. Bevacizumab in combination with oxaliplatin-based chemotherapy as a first-line therapy in metastatic colorectal cancer: a randomized phase III study. J Clin Oncol. 2008;26:1013-9.
57. Mulsow J, Merkel S, Agaimy A, Hohenberger W. Outcomes following surgery for colorectal cancer with synchronous peritoneal metastases. Br J Surg. 2011;98:1785-91.

58. Prada-Villaverde A, Esquivel J, Lowy AM, Markman M, Chua T, Pelz J, et al. American Society of Peritoneal Surface Malignancies evaluation of HIPEC with mitomycin C versus oxaliplatin in 539 patients with colon cancer undergoing a complete cytoreductive surgery. J Surg Oncol. 2014;110:779-85.

59. National Comprehensive Cancer Network. NCCN Guidelines ${ }^{\circledR} \&$ Clinical Resources. https://www.ncen.org/professionals/physician gls/default.aspx. Last accessed May 16, 2018.

60. Alexander HR Jr, Burke AP. Diagnosis and management of patients with malignant peritoneal mesothelioma. J Gastrointest Oncol. 2016;7(1):79-86.

61. Magge D, Zenati MS, Austin F, Mavanur A, Sathaiah M, Ramalingam L, et al. Malignant peritoneal mesothelioma: prognostic factors and oncologic outcome analysis. Ann Surg Oncol. 2014;21:1159-65.

62. Kurman RJ, Shih LM. Molecular pathogenesis and extraovarian origin of ovarian cancer - shifting the paradigm. Hum Path. 2011;42:918-31.

63. Bristow RE, Eisenhauer EL, Santillan A, Chi DS. Delaying the primary surgical effort for advanced ovarian cancer: a systematic review of neoadjuvant chemotherapy and interval cytoreduction. Gynecol Oncol. 2007;104:480-90.

64. Vergote I, Tropé CG, Amant F, Kristensen GB, Ehlen T, Johnson N, European Organization for Research and Treatment of Cancer-Gynaecological Cancer Group, NCIC Clinical Trials Group, et al. Neoadjuvant chemotherapy or primary surgery in stage IIIC or IV ovarian cancer. N Engl J Med. 2010;363:943-53.

65. Karadayi K, Yildiz C, Karakus S, Akkar OB, Ugurlu GP, Kurt A, et al. Cytoreductive surgery and perioperative intraperitoneal chemotherapy for gynecologic malignancies: a single center experience. Eur J Gynaecol Oncol. 2016;37(2):194-8.

66. Jaaback K, Johnson N. Intraperitoneal chemotherapy for the initial management of primary epithelial ovarian cancers. Cochrane Database Syst Rev. 2006;1:CD005340.

67. Helm CW, Richard SD, Pan J, Bartlett D, Goodman MD, Hoefer R, et al. Hyperthermic intraperitoneal chemotherapy in ovarian cancer: first report of the HYPER-O registry. Int J Gynecol Cancer. 2010;20(1):61-9.

68. Deraco M, Virzì S, Iusco DR, Puccio F, Macrì A, Famulari C, et al. Secondary cytoreductive surgery and hyperthermic intraperitoneal chemotherapy for recurrent epithelial ovarian cancer: a multiinstitutional study. BJOG. 2012;119(7):800-9.

69. Deraco M, Kusamura S, Virzì S, Puccio F, Macrì A, Famulari C, et al. Cytoreductive surgery and hyperthermic intraperitoneal chemotherapy as upfront therapy for advanced epithelial ovarian cancer: multi-institutional phase-II trial. Gynecol Oncol. 2011;122(2):215-20.

70. Vaid S, Bell T, Grim R, Ahuja V. Predicting risk of death in general surgery patients on the basis of preoperative variables using American College of Surgeons Quality Improvement Program data. Perm J. 2012;16(4):10-7.

71. Borja-Cacho D, Parsons HM, Habermann EB, Rothenberger DA, Henderson WG, Al-Refaie WB. Assessment of ACS NSQIP/s predictive ability for adverse events after major cancer surgery. Ann Surg Oncol. 2010;17(9):2274-82.

72. Turaga K, Levine E, Barone R, Sticca R, Petrelli N, Lambert $\mathrm{L}$, et al. Consensus guidelines from the American Society of Peritoneal Surface Malignancies on standardizing the delivery of hyperthermic intraperitoneal chemotherapy (HIPEC) in colorectal cancer patients in the United States. Ann Surg Oncol. 2014;21(5):1501-5. 
73. Van Dorp DR, Boston A, Berri RN. Establishing a complex surgical oncology program with low morbidity and mortality at a community hospital. Am J Surg. 2015;209(3):536-41.

74. Low R. Preoperative and surveillance MR imaging of patients undergoing cytoreductive surgery and heated intraperitoneal chemotherapy. J Gastrointest Oncol. 2016;7(1):58-71.

75. Jacquet P, Sugarbaker P. Clinical research methodologies in diagnosis and staging of patients with peritoneal carcinomatosis. Cancer Treat Res. 1996;82:359-74.
76. Low RN, Barone RM, Lee MJ. Surveillance MR imaging is superior to serum tumor markers for detecting early tumor recurrence in patients with appendiceal cancer treated with surgical cytoreduction and HIPEC. Ann Surg Oncol. 2013;20:1074-81.

77. Ford J, Hanna M, Boston A, Berri R. Life after hyperthermic intraperitoneal chemotherapy; measuring quality of life and performance status after cytoreductive surgery plus hyperthermic intraperitoneal chemotherapy. Am J Surg. 2016;211(3):546-50. 